\title{
ALGEBRAIC CHERN-SIMONS THEORY
}

\author{
SPENCER BLOCH AND HÉLÈNE ESNAULT
}

\section{INTRODUCTION}

0.1. Secondary (Chern-Simons) characteristic classes associated to bundles with connection play an important role in differential geometry. We propose to investigate a related construction for algebraic bundles. Non-flat algebraic connections for bundles on complex projective manifolds are virtually non-existent (we know of none), and a deep theorem of Reznikov 18 implies that Chern-Simons classes are torsion for flat bundles on such spaces. On the other hand, it is possible (in several different ways, cf. [1.1] below) given a vector bundle $E$ on $X$ to construct an affine fibration $f: Y \rightarrow X$ (i.e. locally over $X$, $Y \cong X \times \mathbb{A}^{n}$ ) such that $f^{*} E$ admits an algebraic connection. One can arrange moreover that $Y$ itself be an affine variety. Since pullback $f^{*}$ induces an isomorphism from the chow motive of $X$ to that of $Y$, one can in some sense say that every algebraic variety is equivalent to an affine variety, and every vector bundle is equivalent to a vector bundle with an algebraic connection. Thus, an algebraic Chern-Simons theory has some interest. Speaking loosely, the content of such a theory is that a closed differential form $\tau$ representing a characteristic class like the chern class of a vector bundle on a variety $X$ will be Zariski-locally exact, $\tau \mid U_{i}=d \eta_{i}$. The choice of a connection on the bundle enables one to choose the primitives $\eta_{i}$ canonically upto an exact form. In particular, $\left(\eta_{i}-\eta_{j}\right) \mid U_{i} \cap U_{j}$ is exact. When $X$ is affine, a different choice of connection will change the $\eta_{i}$ by a global form $\eta$.

0.2. Unless otherwise noted, all our spaces $X$ will be smooth, quasiprojective varieties over a field $k$ of characteristic 0 . Given a bundle of rank $N$ with connection $(E, \nabla)$ on $X$ and an invariant polynomial $P$ of degree $n$ on the Lie algebra of $\mathrm{GL}_{N}$ (cf. [3]), we construct classes

$$
w_{n}(E, \nabla, P) \in \Gamma\left(X, \Omega_{X}^{2 n-1} / d \Omega_{X}^{2 n-2}\right) ; n \geq 2 .
$$

The first author is supported in part by the NSF, the second one is supported in part by the DFG. 
Here $\Omega_{X}^{i}$ is the Zariski sheaf of Kähler $i$-forms on $X$, and $d: \Omega_{X}^{i} \rightarrow$ $\Omega_{X}^{i+1}$ is exterior differentiation. Zariski locally, these classes are given explicitly in terms of universal polynomials in the connection and its curvature. They satisfy the basic compatibility:

$d w_{n}(E, \nabla, P)$ is a closed $2 n$-form representing the characteristic class in de Rham cohomology associated to $P$ by Chern-Weil theory. Note that $d w_{n}$ is not necessarily exact, because $w_{n}$ is not a globally defined form.

The simplest example is to take $E$ trivial of rank 2 and to assume the connection on the determinant bundle is trivial. The connection is then given by a matrix of 1 -forms $A=\left(\begin{array}{cc}\alpha & \beta \\ \gamma & -\alpha\end{array}\right)$. Taking $P(M)=\operatorname{Tr}\left(M^{2}\right)$ one finds

$$
\begin{gathered}
w_{2}(E, \nabla, P)=2 \alpha \wedge d \alpha-4 \alpha \beta \gamma+\beta d \gamma+\gamma d \beta \\
\text { or, if } A \text { is integrable, } \\
w_{2}(E, \nabla, P)=-2 \alpha \wedge d \alpha=-2 \alpha \beta \gamma .
\end{gathered}
$$

One particularly important invariant polynomial $P_{n}$ maps a diagonal matrix to the $n$-th elementary symmetric function in its entries. We write

$$
w_{n}(E, \nabla):=w_{n}\left(E, \nabla, P_{n}\right)
$$

For example, $P_{2}(M):=\frac{1}{2}(\operatorname{Tr} M)^{2}-\operatorname{Tr}\left(M^{2}\right)$. In fact, when $\nabla$ is integrable, $w_{n}(E, \nabla, P)=\lambda w_{n}(E, \nabla)$ for some coefficient $\lambda \in \mathbb{Q}$ (see 2.3.3).

When $k=\mathbb{C}, w_{n}(E, \nabla)$ is linked to the chern class in $A^{n}(X)$, where $A^{n}(X)$ denotes the group of algebraic cycles modulo a certain adequate equivalence relation, homological equivalence on a divisor. For example, $A^{2}(X)$ is the group of codimension 2 cycles modulo algebraic equivalence. When $n=2$ and $X$ is affine, there is an isomorphism

$$
\varphi: \Gamma\left(X, \Omega_{X}^{3} / d \Omega_{X}^{2}\right) / \Gamma\left(X, \Omega_{X}^{3}\right) \cong A^{2}(X) \otimes_{\mathbb{Z}} \mathbb{C} .
$$

Writing $c_{2, \text { cycle }}(E)$ for the second chern class of $E$ in $A^{2}(X)$, we have

$$
\varphi\left(w_{2}(E, \nabla)\right)=c_{2, \text { cycle }}(E) \otimes 1
$$

0.3. Suppose now the connection $\nabla$ on $E$ is integrable, i.e. $E$ is flat. Let $\mathcal{K}_{i}^{m}$ denote the Zariski sheaf, image of the Zariski Milnor $K$ sheaf in the constant sheaf $K_{i}^{M}(k(X))$. One has a map dlog : $\mathcal{K}_{i}^{m} \rightarrow \Omega_{X, \text { clsd }}^{i}$. Functorial and additive classes

$$
c_{i}(E, \nabla) \in \mathbb{H}^{i}\left(X, \mathcal{K}_{i}^{m} \rightarrow \Omega^{i} \rightarrow \Omega^{i+1} \rightarrow \ldots\right)
$$


were constructed in [8]. One has a natural map of complexes

$$
\sigma:\left\{\mathcal{K}_{i}^{m} \rightarrow \Omega^{i} \rightarrow \Omega^{i+1} \rightarrow \ldots\right\} \rightarrow \Omega_{X}^{2 i-1} / d \Omega_{X}^{2 i-2}[-i] .
$$

We prove in section 4

$$
w_{i}(E, \nabla)=\sigma\left(c_{i}(E, \nabla)\right) \in \Gamma\left(X, \Omega^{2 i-1} / d \Omega^{2 i-2}\right) .
$$

In the case of an integrable connection, the classes $w_{n}(E, \nabla)$ are closed. We are unable to answer the following

0.3.1. basic question. Are the classes $w_{i}(E, \nabla, P)$ all zero for an integrable connection $\nabla$ ?

0.4. We continue to assume $\nabla$ integrable. We take $k=\mathbb{C}$, and $X$ smooth and projective. We define the (generalized) Griffiths group $\operatorname{Griff}^{n}(X)$ to be the group of algebraic cycles of codimension $n$ homologous to zero, modulo those homologous to zero on a divisor. (For $n=2$, this is the usual Griffiths group of codimension 2 algebraic cycles homologous to zero modulo algebraic equivalence.) Our main result is

Theorem 0.4.1. We have $w_{n}(E, \nabla)=0$ if and only if $c_{n}(E)=0$ in $\operatorname{Griff}^{n}(X) \otimes \mathbb{Q}$.

The proof of this theorem is given in section 5 .

The idea is that one can associate to any codimension $n$ cycle $Z$ homologous to zero an extension of mixed Hodge structures of $\mathbb{Q}(0)$ by $H^{2 n-1}(X, \mathbb{Q}(n))$. One gets a quotient extension

$$
0 \rightarrow H^{2 n-1}(X, \mathbb{Q}(n)) / N^{1} \rightarrow E \rightarrow \operatorname{Griff}^{n}(X) \otimes \mathbb{Q}(0) \rightarrow 0
$$

where $N^{1}$ is the subspace of "coniveau" 1 , the group on the right has the trivial Hodge structure and where

$$
E \subset H^{0}\left(X, \mathcal{H}^{2 n-1}(\mathbb{Q}(n))\right) .
$$

Using the classes (0.3.1) and the comparison (0.3.3) we show

$$
w_{n}(E, \nabla) \in F^{0} E \cap E(\mathbb{R}) .
$$

Furthermore, $w_{n}(E, \nabla) \in E(\mathbb{C})$ maps to the class of $c_{n}(E)$. Since the kernel of this extension is pure of weight -1 it follows easily that $w_{n}=0 \Leftrightarrow c_{n}=0$. In fact, Reznikov's theorem [18] implies

$$
w_{n}(E, \nabla) \in E(\mathbb{Q}) .
$$

0.5. Through its link to the Griffiths group, it is clear that the classes $w_{n}(E, \nabla)$, when $\nabla$ is integrable, are rigid in a variation of the flat bundle $(E, \nabla)$ over $X$. But in fact, a stronger rigidity ( see 2.4.1) holds true: one can allow a 1 dimensional variation of $X$ as well. 
0.6. Examples (including Gauß-Manin systems of semi-stable families of curves, weight 1 Gauß-Manin systems, weight 2 Gauß-Manin systems of surfaces, and local systems with finite monodromy) for which the classes $w_{n}(E, \nabla)$ vanish are discussed in section $\square$.

It is possible (cf. section (7) to define $w_{n}(E, \nabla, P)$ in characteristic $p$. In arithmetic situations, the resulting classes are compatible with reduction mod $p$. When the bundle $(E, \nabla)$ in characteristic $p$ comes via Gauß-Manin from a smooth, proper family of schemes over $X$, we show using work of Katz 15 that $w_{n}(E, \nabla, P)=0$. A longstanding conjecture of Ogus [17 would imply that a class in $\Gamma\left(X, \mathcal{H}^{n}\right)$ in characteristic 0, (where $\mathcal{H}$ is the Zariski sheaf of de Rham cohomology), which vanished when reduced $\bmod p$ for almost all $p$ was 0 . Thus, Ogus' conjecture would imply an affirmative answer to 0.3.1 for GaußManin systems.

0.7. In concrete applications, one frequently deals with connections $\nabla$ with logarithmic poles. Insofar as possible, we develop our constructions in this context (see section 6). The most striking remark is that even if $\nabla$ has logarithmic poles, $w_{n}(E, \nabla)$ does not have any poles (see theorem (6.1.1) ).

0.8. Acknowledgments: We thank E. Looijenga for making us aware of the similarity between our invariant $w_{2}$ with Witten's invariant for 3-manifolds (see [20]), and G. van der Geer for communicating [12 to us.

\section{Affine Fibrations}

An affine bundle $Y$ over a scheme $X$ is, by definition, an $\mathcal{V}$-torseur for some vector bundle $\mathcal{V}$. Such things are classified by $H^{1}(X, \mathcal{V})$. In particular, Zariski-locally, $Y \cong X \times \mathbb{A}^{n}$. Pullback from $X$ to $Y$ is an isomorphism on Chow motives, and hence on any Weil cohomology; e.g. $H_{\mathrm{DR}}(X) \cong H_{\mathrm{DR}}(Y), H_{\text {ett }}(X) \cong H_{\text {et }}(Y)$, etc. The following is known as "Jouanolou's trick". We recall the argument from [14].

Proposition 1.0.1. Let $X$ be a quasi-projective variety. Then there exists an affine bundle $Y \rightarrow X$ such that $Y$ is an affine variety.

Proof. Let $X \subset \bar{X}$ be an open immersion with $\bar{X}$ projective. Let $\widetilde{X}$ be the blowup of $\bar{X}-X$ on $\bar{X} \cdot \widetilde{X}$ is projective, and $X \subset \widetilde{X}$ with complement $D$ a Cartier divisor. Suppose we have constructed $\pi: \widetilde{Y} \rightarrow \widetilde{X}$ an affine bundle with $\widetilde{Y}$ affine. Since the complement of a Cartier divisor in an affine variety is affine (the inclusion of the open is acyclic for coherent cohomology, so one can use Serre's criterion) it 
follows that $\pi^{-1}(X) \rightarrow X$ is an affine bundle with $Y:=\pi^{-1}(X)$ affine. We are thus reduced to the case $X$ projective. Let $P(N) \rightarrow \mathbb{P}^{N}$ be an affine bundle with $P(N)$ affine. Given a closed immersion $X \hookrightarrow \mathbb{P}^{N}$, we may pull back $P(N)$ over $X$, so we are reduced to the case $X=\mathbb{P}^{N}$. In this case, one can take $Y=\mathrm{GL}_{N+1} / \mathrm{GL}_{N} \times \mathrm{GL}_{N+1}$.

An exact sequence of vector bundles $0 \rightarrow G \rightarrow F \rightarrow E \rightarrow 0$ on $X$ gives rise to an exact sequence of Hom bundles

$$
0 \rightarrow \operatorname{Hom}(E, G) \rightarrow \operatorname{Hom}(E, F) \rightarrow H o m(E, E) \rightarrow 0
$$

and so an isomorphism class of affine bundles

$$
\partial\left(\operatorname{Id}_{E}\right) \in H^{1}(X, \operatorname{Hom}(E, G)) .
$$

Of particular interest is the Atiyah sequence. Let $X$ be a smooth variety, and let $\mathcal{I} \subset \mathcal{O}_{X} \otimes \mathcal{O}_{X}$ be the ideal of the diagonal. Let $\mathcal{P}_{X}:=$ $\mathcal{O}_{X} \otimes \mathcal{O}_{X} / \mathcal{I}^{2}$, and consider the exact sequence

$$
0 \rightarrow \Omega_{X}^{1} \rightarrow \mathcal{P}_{X} \rightarrow \mathcal{O}_{X} \rightarrow 0
$$

obtained by identifying $\mathcal{I} / \mathcal{I}^{2} \cong \Omega^{1}$ in the usual way. Note that $\mathcal{P}_{X}$ has two distinct $\mathcal{O}_{X}$-module structures, given by multiplication on the left and right. These two structures agree on $\Omega^{1}$ and on $\mathcal{O}_{X}$. Given $E$ a vector bundle on $X$, we consider the sequence (Atiyah sequence)

$$
0 \rightarrow E \otimes_{\mathcal{O}_{X}} \Omega_{X}^{1} \rightarrow E \otimes_{\mathcal{O}_{X}} \mathcal{P}_{X} \rightarrow E \rightarrow 0
$$

The tensor in the middle is taken using the left $\mathcal{O}_{X}$-structure, and then the sequence is viewed as a sequence of $\mathcal{O}_{X}$-modules using the right $\mathcal{O}_{X^{-} \text {-structure. }}$

Proposition 1.0.2. Connections on $E$ are in $1-1$ correspondence with splittings of the Atiyah sequence (1.0.1).

Proof. (See [1] and [5]). As a sequence of sheaves of abelian groups, the Atiyah sequence is split by $e \mapsto e \otimes 1$. Let $\theta: E \rightarrow E \otimes \mathcal{P}_{X}$ be an $\mathcal{O}$-linear splitting. Define

$$
\nabla(e):=\theta(e)-e \otimes 1 \in E \otimes \Omega_{X}^{1} .
$$

We have

$$
\begin{aligned}
& \nabla(f \cdot e):=\theta(e) \cdot(1 \otimes f)-(e \otimes 1)(f \otimes 1)= \\
& \quad(1 \otimes f) \cdot \nabla(e)+(e \otimes 1) \cdot(1 \otimes f-f \otimes 1) \\
& \quad=f \nabla(e)+d f \wedge e
\end{aligned}
$$

which is the connection condition. Conversely, given a connection $\nabla$, the same argument shows that $\theta(e)=\nabla(e)+e \otimes 1$ is an $\mathcal{O}$-linear splitting. 
Corollary 1.0.3. Let $E$ be a vector bundle on a smooth affine variety $X$. Then $E$ admits an algebraic connection.

Proof. An exact sequence of vector bundles on an affine variety admits a splitting.

1.1. In conclusion, given a vector bundle $E$ on a smooth variety $X$, there exists two sorts of affine bundles $\pi: Y \rightarrow X$ such that $\pi^{*} E$ admits a connection. We can take $Y$ to be the Atiyah torseur associated to $E$, in which case the connection is canonical, or we can take $Y$ to be affine, in which case all vector bundles admit (non-canonical) connections.

\section{Chern-Simons}

We begin by recalling in an algebraic context the basic ideas involving connections and the Chern-Weil and Chern-Simons constructions.

2.1. Connections and curvature. Let $R$ be a $k$-algebra of finite type ( $R$ and $k$ commutative with 1 ). A connection $\nabla$ on a module $E$ is a map $\nabla: E \rightarrow E \otimes_{R} \Omega_{R / k}^{1}$ satisfying $\nabla(f \cdot e)=e \otimes d f+f \cdot \nabla e$. More generally, if $D \subset \operatorname{Spec} R$ is a Cartier divisor, of equation $f$, one defines the module $\Omega_{R / k}^{1}(\log D)$ of Kähler 1-forms with logarithmic poles along $D$, as the submodule of forms $w$ with poles along $D$ such that $w \cdot f$ and $w \wedge d f$ are regular [?]. A connection with log poles along $D$ is a $k$ linear map $\nabla: E \rightarrow E \otimes \Omega_{R / k}^{1}(\log D)$ fulfilling the Leibniz relations. When $E$ has a global basis $E=R^{N}, \nabla$ can be written in the form $d+A$, where $A$ is an $N \times N$-matrix of 1 -forms. Writing $e_{i}=(0, \ldots, 1, \ldots, 0)$ we have

$$
\nabla\left(e_{i}\right)=\sum_{j} e_{j} \otimes a_{i j}
$$

The map $\nabla$ extends to a map $\nabla: E \otimes \Omega^{i} \rightarrow E \otimes \Omega^{i+1}$ defined by $\nabla(e \otimes \omega)=\nabla(e) \wedge \omega+e \otimes d \omega$. The curvature of the connection is the map $\nabla^{2}: E \rightarrow E \otimes \Omega^{2}$. The curvature is $R$-linear and is given in the case $E=R^{N}$ by

$$
\begin{aligned}
\nabla^{2}\left(e_{i}\right)=\sum_{j} e_{j} \otimes d a_{i j}+\sum_{j, \ell} e_{\ell} \otimes a_{j \ell} & \wedge a_{i j} \\
= & (0, \ldots, 1, \ldots, 0) \cdot\left(d A-A^{2}\right) .
\end{aligned}
$$

The curvature matrix $F(A)$ is defined by $F(A)=d A-A^{2}$. (Note that the definition $F(A)=d A+A^{2}$ is also found in the literature, e.g. in [3].) 
Given $g \in \mathrm{GL}_{N}(R)$, let $\gamma=g^{-1}$. We can rewrite the connection $\nabla=d+A$ in terms of the basis $\epsilon_{i}:=e_{i} \cdot g=\left(g_{i 1}, \ldots, g_{i N}\right)$, replacing $A$ and $F(A)$ by

$$
\begin{aligned}
d g \cdot g^{-1}+g A g^{-1} & = & -\gamma^{-1} d \gamma+\gamma^{-1} A \gamma \\
F\left(d g \cdot g^{-1}+g A g^{-1}\right) & = & g F(A) g^{-1} .
\end{aligned}
$$

A connection is said to be integrable or flat if $\nabla^{2}=0$. For a connection on $R^{N}$ this is equivalent to $F(A)=0$.

2.2. We recall some basic ideas from [3]. Let $\mathcal{G}$ be a Lie algebra over a field $k$ of characteristic 0 , and let $G$ be the corresponding algebraic group. (The only case we will use is $G=G_{N}$.) Write $\mathcal{G}^{\ell}:=\underbrace{\mathcal{G} \otimes \cdots \otimes \mathcal{G}}_{\ell \text { factors }} . G$ acts diagonally on $\mathcal{G}^{\ell}$ by the adjoint action on each factor, and an element $P$ in the linear dual $\left(\mathcal{G}^{\ell}\right)^{*}$ is said to be invariant if it is invariant under this action. For a $k$-algebra $R$ we consider the module $\Lambda^{r, \ell}:=\mathcal{G}^{\ell} \otimes_{k} \Omega_{R / k}^{r}$ of $r$-forms on $R$ with values in $\mathcal{G}^{\ell}$. Let $x_{i}$ denote tangent vector fields, i.e. elements in the $R$-dual of $\Omega^{1}$. We describe two products $\wedge: \Lambda^{r, \ell} \otimes_{R} \Lambda^{r^{\prime}, \ell^{\prime}} \rightarrow \Lambda^{r+r^{\prime}, \ell+\ell^{\prime}}$ and [ ] : $\Lambda^{r, 1} \otimes_{R} \Lambda^{r^{\prime}, 1} \rightarrow \Lambda^{r+r^{\prime}, 1}$. In terms of values on tangents, these are given by

$$
\begin{gathered}
\varphi \wedge \psi\left(x_{1}, \ldots, x_{r+r^{\prime}}\right)= \\
\sum_{\pi, \text { shuffle }} \sigma(\pi) \varphi\left(x_{\pi_{1}}, \ldots, x_{\pi_{r}}\right) \otimes \psi\left(x_{\pi_{r+1}}, \ldots, x_{\pi_{r+r^{\prime}}}\right) \\
{[\varphi, \psi]\left(x_{1}, \ldots, x_{r+r^{\prime}}\right)=} \\
\sum_{\pi, \text { shuffle }} \sigma(\pi)\left[\varphi\left(x_{\pi_{1}}, \ldots, x_{\pi_{r}}\right), \psi\left(x_{\pi_{r+1}}, \ldots, x_{\pi_{r+r^{\prime}}}\right)\right]
\end{gathered}
$$

Here $\sigma(\pi)$ is the sign of the shuffle. These operations satisfy the identities (for $P \in\left(\mathcal{G}^{\ell}\right)^{*}$ not necessarily invariant)

$$
\begin{aligned}
& {[\varphi, \psi]=\quad(-1)^{r r^{\prime}+1}[\psi, \varphi]} \\
& {[[\varphi, \varphi], \varphi]=\quad 0} \\
& d[\varphi, \psi]=[d \varphi, \psi]+(-1)^{r}[\varphi, d \psi] \\
& d(\varphi \wedge \psi)=d \varphi \wedge \psi+(-1)^{r} \varphi \wedge d \psi \\
& d(P(\varphi))=\quad P(d \varphi) \\
& P(\varphi \wedge \psi \wedge \rho)=(-1)^{r r^{\prime}} P(\psi \wedge \varphi \wedge \rho)
\end{aligned}
$$


If $P$ is invariant, we have in addition for $\varphi_{i} \in \Lambda^{r_{i}, 1}$ and $\psi \in \Lambda^{1,1}$

$$
\sum_{i=1}^{\ell}(-1)^{r_{1}+\ldots+r_{i}} P\left(\varphi_{1} \wedge \cdots \wedge\left[\varphi_{i}, \psi\right] \wedge \cdots \wedge \varphi_{\ell}\right)=0
$$

By way of example, we note that if $A=\left(a_{i j}\right), B=\left(b_{i j}\right)$ are matrices of 1-forms, then writing $A B$ (or $A^{2}$ when $A=B$ ) for the matrix of 2 -forms with entries

$$
\sum_{\ell} a_{i \ell} \wedge b_{\ell j}
$$

we have

$$
\begin{gathered}
{[A, A]\left(x_{1}, x_{2}\right)_{i j}=\left(\left[A\left(x_{1}\right), A\left(x_{2}\right)\right]-\left[A\left(x_{2}\right), A\left(x_{1}\right)\right]\right)_{i j}} \\
=2\left(A\left(x_{1}\right) A\left(x_{2}\right)-A\left(x_{2}\right) A\left(x_{1}\right)\right)_{i j} \\
=2 \sum_{\ell}\left(a_{i \ell}\left(x_{1}\right) a_{\ell j}\left(x_{2}\right)-a_{i \ell}\left(x_{2}\right) a_{\ell j}\left(x_{1}\right)\right) \\
=2 \sum_{\ell} a_{i \ell} \wedge a_{\ell j}\left(x_{1}, x_{2}\right)=2 A^{2}\left(x_{1}, x_{2}\right) .
\end{gathered}
$$

whence

$$
A^{2}=\frac{1}{2}[A, A]
$$

In the following, for $\varphi \in \Lambda^{r, \ell}$ we frequently write $\varphi^{n}$ in place of $\varphi \wedge \ldots \wedge \varphi$ ( $n$-times). The signs differ somewhat from [3] because of our different convention for the curvature as explained above.

Theorem 2.2.1. [3] Let $P \in\left(\mathcal{G}^{\ell}\right)^{*}$ be invariant. To a matrix $A$ of 1 -forms over a ring $R$, we associate a matrix of 2-forms depending on a parameter $t$

$$
\varphi_{t}:=t F(A)-\frac{1}{2}\left(t^{2}-t\right)[A, A]
$$

Define

$$
T P(A)=\ell \int_{0}^{1} P\left(A \wedge \varphi_{t}^{\ell-1}\right) d t \in \Omega_{R / k}^{2 \ell-1}
$$

For example, for

$$
P(M)=\operatorname{Tr} M^{2}, \ell=2, \operatorname{TP}(A)=\operatorname{Tr}\left(A d A-\frac{2}{3} A^{3}\right)
$$

Then $\operatorname{dTP}(A)=P\left(F(A)^{\ell}\right)$. The association $A \mapsto T P(A)$ is functorial for maps of rings $R \rightarrow S$. If $A \mapsto T^{\prime} P(A)$ is another such functorial mapping satisfying

$$
d T^{\prime} P(A)=d T P(A)=P\left(F(A)^{\ell}\right),
$$

then

$$
T^{\prime} P(A)-T P(A)=d \rho
$$


is exact.

Proof. The first assertion follows from Prop. 3.2 of [3], noting that $\Omega(A)$ in their notation is $-F(-A)$ in ours. For the second assertion, we may assume by functoriality that $R$ is a polynomial ring, so $H_{D R}^{2 \ell-1}(R / k)=(0)$. The form $T^{\prime} P(A)-T P(A)$ is closed, and hence exact.

Proposition 2.2.2. With notation as above, let $g \in \mathrm{GL}_{N}(R)$, and assume $\ell \geq 2$. Then $T P\left(d g \cdot g^{-1}+g A g^{-1}\right)-T P(A)$ is Zariski-locally exact, i.e. there exists an open cover $\operatorname{Spec}(R)=\bigcup U_{i}$ such that the above expression is exact on each $U_{i}$.

Proof. The property of being Zariski-locally exact is compatible under pullback, so we may argue universally. The matrix $A$ of 1 -forms (resp. the element $g$ ) is pulled back from the coordinate ring of some affine space $\mathbb{A}^{m}$ (resp. from the universal element in $\mathrm{GL}_{N}$ with coefficients in the coordinate ring of $\mathrm{GL}_{N}$ ), so we may assume $R$ is the coordinate ring of $\mathbb{A}^{m} \times \mathrm{GL}_{N}$.

Let $\eta$ be a closed form on a smooth variety $T$. Let $f: S \rightarrow T$ be surjective, with $S$ quasi-projective. Then $\eta$ is locally exact on $T$ if and only if $f^{*} \eta$ is locally exact on $S$. Indeed, given $t \in T$ we can find a section $S^{\prime \prime} \subset S$ such that the composition $f^{\prime}: S^{\prime} \rightarrow T$, where $S^{\prime} \rightarrow S^{\prime \prime}$ is the normalization, is finite over some neighborhood $t \in U$. Assuming $f^{*} \eta$ is locally exact, it follows that $f^{*} \eta \mid f^{\prime-1}(U)$ is locally exact, and so by a trace argument (we are in characteristic zero) that $\eta \mid U$ is locally exact as well.

We apply the above argument with

$$
\eta=T P\left(d g \cdot g^{-1}+g A g^{-1}\right)-T P(A)
$$

and $T=\mathbb{A}^{m} \times \mathrm{GL}_{N}$. As a scheme, $\mathrm{GL}_{N} \cong \mathbb{G}_{m} \times \mathrm{SL}_{N}$, and for some large integer $M$ we can find a surjection $\coprod_{\text {finite }} \mathbb{A}^{M} \rightarrow \mathrm{SL}_{N}$ by taking products of upper and lower triangular matrices with 1 on the diagonal and then taking a disjoint sum of translates. Pulling back, it suffices to show that a closed form of degree $\geq 2$ on $\mathbb{A}^{M+m} \times \mathbb{G}_{m}$ is exact. This is clear.

2.3. Construction. Let $E$ be a vector bundle of rank $N$ on a smooth quasi-projective variety $X$. Let $P$ be an invariant polynomial as above of degree $n$ on the Lie algebra $\mathcal{G} \mathcal{L}_{N}$. Suppose given a connection $\nabla$ on $E$. (Such a connection exists when $X$ is affine because the Atiyah sequence splits) Let $X=\bigcup U_{i}$ be an open affine covering such that $E \mid U_{i} \cong \mathcal{O}^{\oplus N}$, and let $A_{i}$ be the matrix of 1-forms corresponding to $\nabla \mid U_{i}$. The class of $T P\left(A_{i}\right) \in \Gamma\left(U_{i}, \Omega^{2 n-1} / d \Omega^{2 n-2}\right)$ is independant of 
the choice of basis for $E \mid U_{i}$ by 2.2.2. It follows that these classes glue to give a global class

$$
w_{n}(E, \nabla, P) \in \Gamma\left(X, \Omega^{2 n-1} / d \Omega^{2 n-2}\right)
$$

Proposition 2.3.1. Let $E$ be a rank $N$-vector bundle on a smooth affine variety $X$. Let $\nabla$ and $\nabla^{\prime}$ be two connections on $E$. Let $P$ be an invariant polynomial of degree $n$. Then there exists a form

$$
\eta \in \Gamma\left(X, \Omega_{X}^{2 n-1}\right)
$$

such that

$$
w_{n}(E, \nabla, P)-w_{n}\left(E, \nabla^{\prime}, P\right) \equiv \eta \bmod \left(d \Omega^{2 n-2}\right)
$$

Proof. Because $X$ is affine, any affine space bundle $Y \rightarrow X$ admits a section. (An affine space bundle is a torseur under a vector bundle.) Thus, we may replace $X$ by an affine space bundle over $X$. Since $X$ is affine, $E$ is generated by its global sections, so we may find a Grassmannian $G$ and a map $X \rightarrow G$ such that $E$ is pulled back from $G$. We may find an affine space bundle $Y \rightarrow G$ with $Y$ affine. Replacing $X$ with $X \times_{G} Y$, which is an affine bundle over $X$, we may assume $E$ pulled back from a bundle $F$ on $Y$. Since $Y$ is affine, $F$ admits a connection $\Psi$, and it clearly suffices to prove the proposition for $\nabla$ the pullback of $\Psi$. Write $\nabla^{\prime}-\nabla=\gamma$ with $\gamma \in \operatorname{Hom}_{\mathcal{O}_{X}}\left(E, E \otimes \Omega^{1}\right)$. Let $\iota: X \hookrightarrow \mathbb{A}^{m}$ be a closed immersion. The product map $X \hookrightarrow Y \times \mathbb{A}^{m}$ is a closed immersion, hence $\gamma$ lifts to $\varphi \in \operatorname{Hom}_{\mathcal{O}_{Y \times \mathbb{A}}}\left(F, F \otimes \Omega_{Y \times \mathbb{A}^{m}}^{1}\right)$. Let $\Psi^{\prime}:=\Psi+\varphi$. We are now reduced to the case $X=Y \times \mathbb{A}^{m}$. Writing $\mathcal{H}^{2 n-1}$ for the Zariski cohomology sheaf of the de Rham complex on $X$, one knows that $\Gamma\left(X, \mathcal{H}^{2 n-1}\right) \subset \Gamma\left(U, \mathcal{H}^{2 n-1}\right)$ for any open $U \neq \emptyset$ [2]. Taking $U=\mathbb{A}^{M+m}$, where $\mathbb{A}^{M}$ is an affine cell in $Y$, we may assume $\Gamma\left(X, \mathcal{H}^{2 n-1}\right)=(0)$. Now $d w(E, \nabla, P)$ and $d w\left(E, \nabla^{\prime}, P\right)$ both represent the same class in cohomology, so, since $X$ is affine, there exists $\eta \in \Gamma\left(X, \Omega^{2 n-1}\right)$ such that

$$
w_{n}(E, \nabla, P)-w_{n}\left(E, \nabla^{\prime}, P\right)-\eta \in \Gamma\left(X, \mathcal{H}^{2 n-1}\right)=(0) .
$$

Proposition 2.3.2. Let $\nabla$ be an integrable connection on $E$, and let $P$ be an invariant polynomial of degree $n$. Let $\mathcal{H}^{2 n-1}=\Omega_{\text {closed }}^{2 n-1} / d \Omega^{2 n-2}$. Then $w_{n}(E, \nabla, P) \in \Gamma\left(X, \mathcal{H}^{2 n-1}\right)$, i.e. $d w=0$.

Proof. $d w=P(F(\nabla))=0$ since $\nabla$ integrable implies $F(\nabla)=0$. 
Proposition 2.3.3. Let $\nabla$ be an integrable connection on $E$, and let $P=\lambda P_{n}+Q$ be an invariant polynomial of degree $n$, where $P_{n}$ is the $n$-th elementary symmetric function and

$$
Q=\sum \mu_{i j} P_{i} \cdot P_{j}, i \neq j, i \geq 1, j \geq 1, \lambda \in \mathbb{Q}, \mu_{i j} \in \mathbb{Q} .
$$

Then

$$
w_{n}(E, \nabla, P)=\lambda w_{n}(E, \nabla)
$$

(see notation (0.2.3)).

Proof. It is enough to see that $w_{n}\left(E, \nabla, P_{i} \cdot P_{j}\right)=0$ for $i \geq 1, j \geq 1$. One has $P_{j}\left(F(A)^{j}\right)=0$, so by Theorem 2.2 .1

$$
T^{\prime}\left(P_{i} \cdot P_{j}\right)(A)=T P_{i}(A) \cdot P_{j}\left(\left(F\left(A_{j}\right)\right)^{j}\right)=0
$$

differs from $T\left(P_{i} \cdot P_{j}\right)(A)$ by an exact form on the open on which $A$ is defined.

\subsection{Rigidity.}

Theorem 2.4.1. Let $f: X \rightarrow S$ be a smooth proper morphism between smooth algebraic varieties defined over a field $k$ of characteristic zero. Assume $\operatorname{dim} S=1$. Let $\nabla: E \rightarrow \Omega_{X / S}^{1} \otimes E$ be a relative flat connection, and $P$ be an invariant polynomial. Then

$$
w_{n}(E, \nabla, P) \in H^{0}\left(X, \mathcal{H}^{2 n-1}(X / S)\right)
$$

lifts canonically to a class in $H^{0}\left(X, \mathcal{H}^{2 n-1}\right)$ for $n \geq 2$.

Proof. Take locally the matrix $A_{i}^{\prime} \in H^{0}\left(X_{i}, M\left(N, \Omega_{X / S}^{1}\right)\right)$ of the connection, $N$ being the rank of $E$. Take liftings $A_{i} \in H^{0}\left(X_{i}, M\left(N, \Omega_{X}^{1}\right)\right)$, and define $T P\left(A_{i}\right)$ looking at the $\Omega_{X}^{1}$ valued connection defined by $A_{i}$. Since $F\left(A_{i}\right) \in H^{0}\left(X_{i}, M\left(N, f^{*} \Omega_{S}^{1}\right)\right)$, one has $F\left(A_{i}\right)^{n}=0$ for $n \geq 2$, and $d T P\left(A_{i}\right)=P\left(F(A)^{n}\right)=0$. On $X_{i} \cap X_{j}$, one has

$$
A_{j}=d g \cdot g^{-1}+g A_{i} g^{-1}-\Gamma_{i j}
$$

where $\Gamma_{i j} \in H^{0}\left(X_{i} \cap X_{j}, f^{*} \Omega_{S}^{1}\right)$. Using proposition 2.2.2, we just have to show that $T P(B)-T P(B+\Gamma)$ is locally exact for some matrix of one forms $B=d g \cdot g^{-1}+g A_{i} g^{-1}$, verifying $F(B) \omega=0$ for any $w \in M\left(N, f^{*} \Omega_{S}^{1}\right)$, and $\Gamma=\Gamma_{i j} \in f^{*} \Omega_{S}^{1}$. By 2.3.3 it is enough to consider $P(M)=\operatorname{Tr} M^{n}$. One has

$$
\varphi_{t}(B+\Gamma)=F(t(B+\Gamma))=F(t B)+t d \Gamma-t^{2}(\Gamma B+B \Gamma)
$$

and

$$
F(t B) \omega=\left(t-t^{2}\right) d B \omega
$$


with $\omega$ as above. Thus

$$
P\left((B+\Gamma) \wedge \varphi_{t}^{n-1}(B+\Gamma)\right)=
$$

$$
\begin{gathered}
\operatorname{Tr}(B+\Gamma)\left[\left(t d B-t^{2} B^{2}\right)^{n-1}+(n-1)\left(t-t^{2}\right)^{n-2}(d B)^{n-2}\left(t d \Gamma-t^{2}(B \Gamma+\Gamma B)\right]\right. \\
=P\left(B \wedge \varphi_{t}^{n-1}(B)\right)+R
\end{gathered}
$$

with

$$
\begin{aligned}
& R=\operatorname{Tr} \Gamma(d B)^{n-1}\left[\left(t-t^{2}\right)^{n-1}-2 t^{2}(n-1)\left(t-t^{2}\right)^{n-2}\right] \\
&+(n-1)\left(t-t^{2}\right)^{n-2} t \operatorname{Tr} B(d B)^{n-2} d \Gamma
\end{aligned}
$$

Write $\operatorname{Tr} d(B \Gamma)=\operatorname{Tr} d B \Gamma-\operatorname{Tr} B d \Gamma$. Then we have

$$
R=F(t) \operatorname{Tr} \Gamma(d B)^{n-1}
$$

modulo exact forms, with

$$
F(t)=n\left(t-t^{2}\right)^{n-1}-(n-1) t^{2}\left(t-t^{2}\right)^{n-2}=\left(t\left(t-t^{2}\right)^{n-1}\right)^{\prime} .
$$

The assertion now follows from (2.2.10).

\section{Flat Bundles}

The following notations will reoccur frequently.

3.1. $X$ will be a smooth variety, and $D=\bigcup D_{i} \subset X$ will be a normal crossings divisor, with $j: X-D \rightarrow X$. We will assume unless otherwise specified that the ground field $k$ has characteristic 0 .

3.2. $(E, \nabla)$ will be a vector bundle $E$ of rank $r$ on $X$ with connection $\nabla: E \rightarrow E \otimes \Omega_{X / k}^{1}(\log D)$ having logarithmic poles along $D$. The Poincaré residue map $\Omega^{1}(\log D) \rightarrow \mathcal{O}_{D_{i}}$ is denoted $\operatorname{res}_{D_{i}}$, and $\Gamma_{i}:=$ $\operatorname{res}_{D_{i}} \circ \nabla:\left.E \rightarrow E\right|_{D_{i}}$.

3.3. When $E$ is trivialized on the open cover $X=\cup X_{i}$, with basis $\underline{e}_{i}$ on $X_{i}$, then $(E, \nabla)$ is equivalent to the data

$$
\begin{aligned}
g_{i j} & \in \Gamma\left(X_{i} \cap X_{j}, G L\left(r, \mathcal{O}_{X}\right)\right) \\
g_{i k} & =g_{i j} g_{j k} \\
A_{i} & \in \Gamma\left(X_{i}, M\left(r, \Omega_{X}^{1}(\log D)\right)\right)
\end{aligned}
$$

with $g_{i j}^{-1} d g_{i j}=g_{i j}^{-1} A_{i} g_{i j}-A_{j}$.

3.4. The curvature

$$
\nabla^{2}: E \rightarrow \Omega_{X}^{2}(\log D) \otimes E
$$

is given locally by

$$
\nabla^{2}=F\left(A_{i}\right):=d A_{i}-A_{i} A_{i}
$$

The connection $\nabla$ is said to be flat, or integrable if $\nabla^{2}=0$. 
3.5. For two $r \times r$ matrices $A$ and $B$ of differential forms of weight $a$ and $b$ respectively, one writes $\operatorname{Tr} A B$ for the trace of the $r \times r$ matrix $A B$ of weight $a+b$, and one has $\operatorname{Tr} A B=(-1)^{a b} \operatorname{Tr} B A$. We denote by ${ }^{t} A$ the transpose of $A:\left({ }^{t} A\right)_{i j}=A_{j i}$.

3.6. For any cohomology theory $H$ with a localization sequence, the $i$-th level of Grothendieck's coniveau filtration is defined by

$$
\begin{aligned}
N^{i} H^{\bullet}=\left\{x \in H^{\bullet} \mid \exists \text { subvariety } Z \subset X \text { of codimension } \geq i\right. \\
\text { such that } 0=\left.x\right|_{X-Z} \in H^{\bullet}(X-Z) .
\end{aligned}
$$

3.7. For any cohomology theory $H$ defined in a topology finer than the Zariski topology, one defines the Zariski sheaves $\mathcal{H}$ associated to the presheaves $U \mapsto H(U)([2])$. When $H$ is the cohomology for the analytic topology with coefficients in a constant sheaf $A$, we sometimes write $\mathcal{H}(A)$. For example the Betti or de Rham sheaves $\mathcal{H}(\mathbb{C})$ are simply the cohomology sheaves for the complex of algebraic differentials $\Omega_{X}^{*}$. For $D \subset X$ as above, we write $\mathcal{H}^{\bullet}(\log D)$ for the cohomology sheaves of $\Omega^{*}(\log D)$. It is known that $\mathcal{H}^{\bullet}(\log D) \cong j_{*} \mathcal{H}_{X-D}$.

3.8. When $k=\mathbb{C}$ we use the same notation $\Omega_{X}^{*}$ for the analytic and algebraic de Rham complexes. For integers $a$ and $b$, the analytic Deligne cohomology is defined to be the hypercohomology of the complex of analytic sheaves

$$
H_{\mathcal{D}, \text { an }}^{a}(X, \mathbb{Z}(b)):=\mathbb{H}^{a}\left(X_{\text {an }}, \mathbb{Z}(b) \rightarrow \mathcal{O} \rightarrow \Omega^{1} \rightarrow \cdots \rightarrow \Omega^{b-1}\right) .
$$

(This should be distinguished from the usual Deligne cohomology, which is defined using differentials with at worst log poles at infinity.) One has a cycle class map from the Chow group of algebraic cycles modulo rational equivalence to Deligne cohomology:

$$
C H^{i}(X) \rightarrow H_{\mathcal{D}, \text { an }}^{2 i}(X, \mathbb{Z}(i)) .
$$

3.9. We continue to assume $k=\mathbb{C}$. Let $\alpha: X_{\text {an }} \rightarrow X_{\text {Zar }}$ be the identity map. For a complex $C$, let $t_{\geq i} C$ be the subcomplex which is zero in degrees $<i$ and coincides with $C$ in degrees $\geq i$. There is a map of complexes $t_{\geq i} C \cdot \rightarrow$. The complex

$$
\mathbb{Z}(j) \rightarrow \mathcal{O}_{X} \rightarrow \Omega_{X}^{1} \rightarrow \cdots
$$

in the analytic topology is quasi-isomorphic to the cone $\mathbb{Z}(j) \rightarrow \mathbb{C}$, and hence to $\mathbb{C} / \mathbb{Z}(j)[-1]$. We obtain in this way a map in the derived category

$$
\left(t_{\geq j} \Omega_{X}\right) \rightarrow \mathbb{C} / \mathbb{Z}(j)
$$


The kernel of the resulting map

$$
R^{j} \alpha_{*}\left(t_{\geq j} \Omega_{X}\right) \cong \operatorname{ker}\left(\alpha_{*} \Omega^{j} \rightarrow \alpha_{*} \Omega^{j+1}\right) \rightarrow R^{j} \alpha_{*}(\mathbb{C} / \mathbb{Z}(j))
$$

is denoted $\Omega_{\mathbb{Z}(j)}^{j}$ [8]. Note $\Omega_{\mathbb{Z}(j)}^{j}$ is a Zariski sheaf. Writing $\mathcal{K}_{j}^{m}$ for the Milnor $K$-sheaf (subsheaf of the constant sheaf $K_{j}^{\text {Milnor }}(k(X))$ ), the $d \log$-map

$$
\left\{f_{1}, \ldots, f_{j}\right\} \mapsto d f_{1} / f_{1} \wedge \cdots \wedge d f_{j} / f_{j}
$$

induces a map

$$
d \log : \mathcal{K}_{j}^{m} \rightarrow \Omega_{\mathbb{Z}(j)}^{j}
$$

To see this, note the exponential sequence induces a map

$$
\mathcal{O}_{X_{\text {Zar }}}^{*} \rightarrow R^{1} \alpha_{*} \mathbb{Z}(1)
$$

and we get by cup product a commutative diagram with left hand vertical arrow surjective

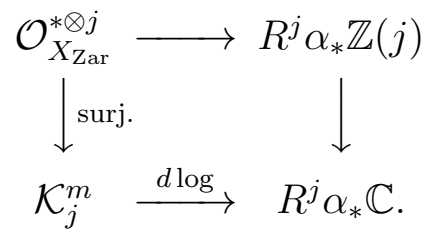

We shall need some more precise results about the sheaf $\Omega_{\mathbb{Z}(j)}^{j}$.

Lemma 3.9.1. $\quad$ 1. There is a natural map

$$
H^{i}\left(X_{\mathrm{Zar}}, \Omega_{\mathbb{Z}(i)}^{i}\right) \rightarrow H_{\mathcal{D}, \text { an }}^{2 i}(X, \mathbb{Z}(i))
$$

2. Let $D \subset X$ be a normal crossings divisor. Then there is a natural map

$$
\begin{aligned}
& \mathbb{H}^{i}\left(X_{\mathrm{Zar}}, \Omega_{\mathbb{Z}(i)}^{i} \rightarrow \alpha_{*} \Omega_{X}^{i}(\log D) \rightarrow \ldots\right) \rightarrow \\
& \quad \mathbb{H}^{2 i}\left(X_{\mathrm{an}}, \mathbb{Z}(i) \rightarrow \mathcal{O}_{X} \rightarrow \Omega_{X}^{1} \rightarrow \ldots \rightarrow \Omega_{X}^{i-1} \rightarrow \Omega^{i}(\log D)_{X} \rightarrow \ldots\right)
\end{aligned}
$$

3. There is a natural map

$$
\begin{aligned}
\varphi & : \mathbb{H}^{i}\left(X_{\text {Zar }}, \mathcal{K}_{i}^{m} \stackrel{d \log }{\rightarrow} \Omega_{X}^{i}(\log D) \rightarrow \ldots\right) \rightarrow \\
& \mathbb{H}^{2 i}\left(X_{\mathrm{an}}, \mathbb{Z}(i) \rightarrow \mathcal{O}_{X} \rightarrow \Omega_{X}^{1} \rightarrow \ldots \rightarrow \Omega_{X}^{i-1} \rightarrow \Omega^{i}(\log D)_{X} \rightarrow \ldots\right)
\end{aligned}
$$

In particular, for $D=\emptyset$, we get a map

$$
\mathbb{H}^{i}\left(X_{\mathrm{Zar}}, \mathcal{K}_{i}^{m} \stackrel{d \log }{\longrightarrow} \Omega_{X}^{i} \rightarrow \ldots\right) \rightarrow H^{2 i-1}\left(X_{\mathrm{an}}, \mathbb{C} / \mathbb{Z}(i)\right) .
$$

Proof. We consider the spectral sequence

$$
\begin{gathered}
R^{j}:=R^{j} \alpha_{*}\left(\mathbb{Z}(i) \rightarrow \mathcal{O} \rightarrow \Omega^{1} \rightarrow \ldots \rightarrow \Omega^{i-1}\right) \\
E_{2}^{p, q}=H^{p}\left(X_{\text {Zar }}, R^{q}\right) \Rightarrow H_{\mathcal{D}, \text { an }}^{p+q}(X, \mathbb{Z}(i))
\end{gathered}
$$


One checks that

$$
\begin{gathered}
R^{s} \cong \mathcal{H}^{s-1}(\mathbb{C} / \mathbb{Z}(i)) ; \quad s<i \\
0 \rightarrow \mathcal{H}^{i-1}(\mathbb{C} / \mathbb{Z}(i)) \rightarrow R^{i} \rightarrow \Omega_{\mathbb{Z}(i)}^{i} \rightarrow 0 \\
0 \rightarrow \mathcal{H}^{i-1}(\mathbb{C} / \mathbb{Z}(i)) \rightarrow R^{s} \rightarrow \operatorname{ker}\left(\mathcal{H}^{s}(\mathbb{C}) \rightarrow \mathcal{H}^{s}(\mathbb{C} / \mathbb{Z}(i))\right) \rightarrow 0 ; \quad s>i
\end{gathered}
$$

We have by ([2]) that $H^{a}\left(X_{\mathrm{Zar}}, \mathcal{H}^{b}(A)\right)=(0)$ for $a>b$ and $A$ any constant sheaf of abelian groups. Applying this to the above, we conclude $E_{2}^{a, 2 i-a}=H^{a}\left(X_{\mathrm{Zar}}, R^{2 i-a}\right)=(0)$ for $a>i$, and $E_{2}^{i, i} \cong H^{i}\left(X, \Omega_{\mathbb{Z}(i)}^{i}\right)$. Assertion (11) follows. The construction of the map in (2) is similar and is left for the reader. Finally, (3) follows by composing the arrow from (2) with the $d$ log map 3.9.1.

3.10. Characteristic classes. Let $(E, \nabla)$ be a bundle with connection as in 3.2 and assume $\nabla$ is flat. Functorial and additive characteristic classes

$$
c_{i}(E, \nabla) \in \mathbb{H}^{i}\left(X_{\text {Zar }}, \mathcal{K}_{i}^{m} \rightarrow \Omega_{X}^{i}(\log D) \rightarrow \Omega_{X}^{i+1}(\log D) \rightarrow \ldots\right)
$$

were defined in [7]. These classes have the following compatibilities:

3.10.1. Under the map

$$
\begin{aligned}
\mathbb{H}^{i}\left(X_{\text {Zar }}, \mathcal{K}_{i}^{m} \rightarrow \Omega_{X}^{i}(\log D) \rightarrow \Omega_{X}^{i+1}(\log D) \rightarrow \ldots\right) \rightarrow & \\
& H^{i}\left(X, \mathcal{K}_{i}^{m}\right) \cong C H^{i}(X)
\end{aligned}
$$

we have $c_{i}(E, \nabla) \mapsto c_{i}^{\text {Chow }}(E) \in C H^{i}(X)$.

3.10.2. Assume $X$ proper and $D=\phi$. The classes $c_{i}(E, \nabla)$ lift classes $c_{i}^{\text {an }}(E, \nabla) \in H^{2 i-1}\left(X_{\text {an }}, \mathbb{C} / \mathbb{Z}(i)\right)$ defined in [8], via the commutative diagram

$$
\begin{array}{cc}
\mathbb{H}^{i}\left(X, \mathcal{K}_{i}^{m} \rightarrow \Omega_{X}^{i} \rightarrow \Omega_{X}^{i+1} \rightarrow \ldots\right) & \longrightarrow C H^{i}(X) \\
\varphi \sqrt[3.9 .1]{(3))} \mid & \mid \downarrow=\text { cycle map } \\
H^{2 i-1}\left(X_{\mathrm{an}}, \mathbb{C} / \mathbb{Z}(i)\right) & \longrightarrow H_{\mathcal{D}}^{2 i}(X, i) .
\end{array}
$$

3.10.3. When $D \neq \phi$ and $X$ is proper, classes

$$
c_{i}^{\text {an }}(E, \nabla) \in \mathbb{H}^{2 i}\left(X_{\text {an }}, \mathbb{Z}(i) \rightarrow \mathcal{O}_{X} \rightarrow \ldots \rightarrow \Omega_{X}^{i-1} \rightarrow \Omega_{X}^{i}(\log D) \rightarrow \ldots\right)
$$

lifting $c_{i}^{\mathcal{D}}(E) \in H_{\mathcal{D}}^{2 i}(X, \mathbb{Z}(i))$ are defined in $[8$. In general, for $X$ not proper, these classes lift

$$
c_{i}^{\mathcal{D}}\left(\left.E\right|_{X-D}\right) \in H_{\mathcal{D}}^{2 i}(X-D, \mathbb{Z}(i))
$$

via the factorization through $H^{2 i-1}(X-D, \mathbb{C} / \mathbb{Z}(i))([9],(3.5))$. 
Proposition 3.10.1. The map $\varphi$ from $3.9 .1(3))$ carries $c_{i}(E, \nabla)$ to $c_{i}^{\text {an }}(E, \nabla)$. For $X$ proper, the diagram

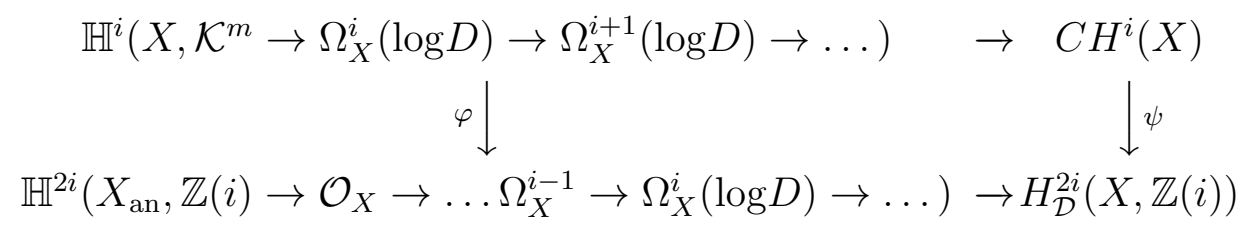

commutes. For $X$ not proper, the diagram remains commutative if one replaces the bottom row by

$$
H^{2 i-1}\left((X-D)_{\mathrm{an}}, \mathbb{C} / \mathbb{Z}(i)\right) \rightarrow H_{\mathcal{D}}^{2 i}(X-D, \mathbb{Z}(i))
$$

or if one replaces $H_{\mathcal{D}}^{2 i}(X, \mathbb{Z}(i))$ by $H_{\mathcal{D}, \text { an }}^{2 i}(X, \mathbb{Z}(i))$.

Proof. The central point, for which we refer the reader to ([8]) is the following. Let $\pi: G \rightarrow X$ be the flag bundle of $E$ over which $E$ has a filtration $E_{i-1} \subset E_{i}$ by $\tau \nabla$ stable subbundles with successive rank 1 quotients $\left(L_{i}, \tau \nabla\right)$ (see [7]). Then $c_{i}(E, \nabla)$ and $c_{i}^{\text {an }}(E, \nabla)$ are both defined on $G$ by products starting from

$$
\begin{gathered}
c_{1}\left(L_{\alpha}, \tau \nabla\right) \in \mathbb{H}^{1}\left(G, \mathcal{K}_{1} \rightarrow \pi^{*} \Omega_{X}^{1}(\log D) \rightarrow \ldots\right) \\
c_{1}^{\mathrm{an}}\left(L_{\alpha}, \tau \nabla\right) \in \mathbb{H}^{2}\left(G, \mathbb{Z}(i) \rightarrow \mathcal{O}_{G} \rightarrow \pi^{*} \Omega_{X}^{1}(\log D) \rightarrow \ldots\right) .
\end{gathered}
$$

It suffices to observe that the "algebraic" product

$$
\begin{aligned}
\mathbb{H}^{1}\left(G, \mathcal{K}_{1} \rightarrow \pi^{*} \Omega_{X}^{1}(\log D) \rightarrow\right. & \ldots)^{\otimes i} \\
& \rightarrow \mathbb{H}^{i}\left(G, \mathcal{K}_{i}^{m} \rightarrow \pi^{*} \Omega_{X}^{i}(\log D) \rightarrow \ldots\right)
\end{aligned}
$$

([8], p. 51) is defined compatibily with the "analytic" product

$$
\begin{aligned}
\mathbb{H}^{2}(G, & \left.\mathbb{Z}(i) \rightarrow \mathcal{O}_{G} \rightarrow \pi^{*} \Omega_{X}^{1}(\log D) \rightarrow \ldots\right)^{\otimes i} \\
& \rightarrow \mathbb{H}^{2 i}\left(G, \mathbb{Z}(i) \rightarrow \mathcal{O}_{G} \rightarrow \ldots \rightarrow \Omega_{G}^{i-1} \rightarrow \pi^{*} \Omega_{X}^{i}(\log D) \rightarrow \ldots\right)
\end{aligned}
$$

3.11. Let $\tau: \Omega_{X}^{*} \rightarrow N^{*}$ be a map of complexes, with $\mathcal{O}_{X}=N^{0}$, such that if $a$ is the smallest degree $b$ for which $B^{b}:=\operatorname{Ker} \Omega_{X}^{b} \rightarrow N^{b} \neq 0$, then $B^{b}=B^{a} \wedge \Omega_{X}^{b-a}$. For example, let $\nabla: \mathcal{F} \rightarrow \Omega_{X}^{1}(\log D) \otimes \mathcal{F}$ be a non integrable connection. Then the local relation $d F(A)=[A, F(A)]$ shows that one can define $N^{*}$ by

$$
\begin{gathered}
N^{1}=\Omega_{X}^{1}(\log D) \\
N^{i}=\Omega_{X}^{i}(\log D) / B^{2} \wedge \Omega_{X}^{i-2}(\log D)
\end{gathered}
$$


where $B^{2}$ is locally generated by the entries of the curvature matrix of $\nabla$.

Let $(E, \nabla)$ be a flat $N^{*}$ valued connection, that is a $k$ linear map $\nabla: E \rightarrow N^{1} \otimes E$ satisfying the Leibniz rule

$$
\nabla(\lambda e)=\tau d \lambda e+\lambda \nabla(e)
$$

the sign convention

$$
\nabla(\omega \otimes e)=\tau d(\omega) \otimes e+(-1)^{o} \omega \wedge \nabla(e),
$$

where $o=\operatorname{deg} \omega$, and $(\nabla)^{2}=0$. Then the computations of [7] and [8] allow to show the existence of functorial and additive classes

$$
c_{i}(E, \nabla) \in \mathbb{H}^{i}\left(X, \mathcal{K}_{i}^{m} \rightarrow N^{i} \rightarrow N^{i+1} \ldots\right)
$$

mapping to analytic classes

$$
c_{i}^{\mathrm{an}}(E, \nabla) \in \mathbb{H}^{2 i}\left(X_{\mathrm{an}}, \mathbb{Z}(i) \rightarrow \ldots \rightarrow \Omega_{X}^{i} \rightarrow N^{i+1} . .\right)
$$

compatibly with the classes $c_{i}^{\mathcal{D}}(E)$ and $c_{i}^{\text {Chow }}(E)$ as before. As we won't need those classes, we don't repeat in details the construction.

3.12. Finally, the $c_{i}(E, \nabla)$ map to classes

$$
\theta_{i}(E, \nabla) \in H^{0}\left(X, \frac{\Omega_{X}^{2 i-1}(\log D)}{d \Omega_{X}^{2 i-2}(\log D)}\right) .
$$

In the next section these will be related to the classes $w_{i}(E, \nabla)$.

\section{THE CLASSES $\theta_{n}$ AND $w_{n}$}

Recall that we had defined $w_{n}(E, \nabla)=w_{n}\left(E, \nabla, P_{n}\right)$ in $(0.2 .3)$ for the $n$-th elementary symmetric function $P_{n}$.

Theorem 4.0.1. Let $X$ be a smooth quasi-projective variety over $\mathbb{C}$. Let $E, \nabla$ be a rank $d$ vector bundle on $X$ with integrable connection. For $d \geq n \geq 2$, we have $w_{n}(E, \nabla)=\theta_{n}(E, \nabla)$, and $w_{n}(E, \nabla, P)=$ $\lambda \theta_{n}(E, \nabla)$ (with the notations of Proposition 2.3.2).

The proof will take up this entire section. We begin with

Remark 4.0.2. We may assume $X$ is affine, and $E \cong \mathcal{O}_{X}^{\oplus N}$. In this situation, the class $w_{n}(E, \nabla)$ lifts canonically to a class in $H^{0}\left(X, \Omega^{2 n-1}\right) / d H^{0}\left(X, \Omega^{2 n-2}\right)$.

Indeed, one knows from [2] that for $U \subset X$ non-empty open, the restriction map $H^{0}\left(X, \mathcal{H}^{2 n-1}\right) \rightarrow H^{0}\left(U, \mathcal{H}^{2 n-1}\right)$ is injective. The assertion about lifting follows from the construction of $w_{n}$ in section 2 because the trivialization can be taken globally. 
4.1. The connection is now given by a matrix of 1-forms and so can be pulled back in many ways from some (non-integrable) connection $\Psi$ on the trivial bundle $\mathcal{E} \cong \mathcal{O}_{\AA^{p}}^{N}$. We will want to assume $\Psi$ "general" in a sense to be specified below. For convenience, write $T=\mathbb{A}^{p}$ and let $\varphi: X \rightarrow T$ be the map pulling back the connection. Let $\pi: P \rightarrow T$ be the flag bundle for $\mathcal{E}$ and let $Q=\varphi^{*} P$, so we get a diagram

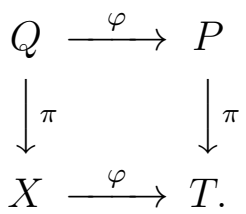

4.2. The curvature $F(\Psi)$ defines an $\mathcal{O}_{T}$-linear map

$$
F(\Psi): \mathcal{E} \rightarrow \mathcal{E} \otimes_{\mathcal{O}_{T}} \Omega_{T}^{2}
$$

In concrete terms, we take $p=2 N^{2} q$ for some large integer $q$, and we write $x_{i j}^{(k)}$ and $y_{i j}^{(k)}$ for $1 \leq i, j \leq N$ and $1 \leq k \leq q$ for the coordinates on $\mathbb{A}^{p}$. The connection $\Psi$ then corresponds to an $N \times N$ matrix of 1 -forms $A=\left(a_{i j}\right)$, and the curvature is given by $F(\Psi):=\left(f_{i j}\right)=d A-A^{2}$. We take

$$
a_{i j}=\sum_{\ell=1}^{q} x_{i j}^{(\ell)} d y_{i j}^{(\ell)} ; \quad f_{i j}=d a_{i j}-\sum_{m=1}^{N} a_{i m} \wedge a_{m j} .
$$

Notice that for $q$ large, we can find $\varphi: X \rightarrow T$ so that $(\mathcal{E}, \Psi)$ pulls back to $(E, \nabla)$.

4.3. We want to argue universally by computing characteristic classes for $(\mathcal{E}, \Psi)$, but the curvature gets in the way. We could try to kill the curvature and look for classes in the quotient complex of $\Omega_{T}^{*}$ modulo the differential ideal generated by the $f_{i j}$ (see 3.11), but this gratuitous violence seems to lead to difficulties. Instead, we will use the notion of $\tau$-connection defined in [7] and [8] and work with a sheaf of differential algebras

$$
M^{*}=\Omega_{P}^{*} / \mathcal{I}
$$

on the flag bundle $P$.

Let

$$
\mu: \Omega_{P / T}^{1} \stackrel{\iota}{\longrightarrow} \pi^{*} \operatorname{Hom}(\mathcal{E}, \mathcal{E}) \stackrel{\pi^{*} F(\Psi)}{\longrightarrow} \pi^{*} \Omega_{T}^{2}
$$

be the composition, where $\iota$ is the standard inclusion on a flag bundle. An easy way to see $\iota$ is to consider the fibration $R \rightarrow P=R / B$, where $R$ is the corresponding principal $G=G L(N)$ bundle and $B$ is the Borel subgroup of upper triangular matrices, and to write the surjection $\mathcal{T}(R / T) / B \rightarrow \mathcal{T}(P / T)$ dual to $\iota$, where $\mathcal{T}(A / B)$ is the relative tangent 
space of $A$ with respect to $B$. There is an induced map of graded $\pi^{*} \Omega_{T^{-}}^{*}$ modules, and we define the graded algebra $M^{*}$ to be the cokernel as indicated:

$$
\Omega_{P / T}^{1} \otimes_{\mathcal{O}_{P}} \pi^{*} \Omega_{T}^{*}[-2] \stackrel{\mu \otimes 1}{\longrightarrow} \pi^{*} \Omega_{T}^{*} \rightarrow M^{*} \rightarrow 0 .
$$

Note $M^{0}=\mathcal{O}_{P}$ and $M^{1}=\pi^{*} \Omega_{T}^{1}$.

Proposition 4.4.1. (i) Associated to the connection $\Psi$ on $\mathcal{E}$ there is an $\mathcal{O}_{P}$-linear splitting $\tau: \Omega_{P}^{1} \rightarrow \pi^{*} \Omega_{T}^{1}$ of the natural inclusion $\pi^{*} \Omega_{T}^{1} \stackrel{i}{\longrightarrow} \Omega_{P}^{1}$. The resulting map $\delta:=\tau \circ d: \mathcal{O}_{P} \rightarrow \pi^{*} \Omega_{T}^{1}$ is a derivation, which coincides with the exterior derivative on $\pi^{-1} \mathcal{O}_{T} \subset \mathcal{O}_{P}$. By extension, one defines

$$
\delta: \pi^{*} \Omega_{T}^{n} \rightarrow \pi^{*} \Omega_{T}^{n+1} ; \quad \delta\left(f \pi^{-1} \omega\right)=f \pi^{-1} d \omega+\delta(f) \wedge \pi^{-1} \omega
$$

(ii) One has

$$
\delta^{2}=\mu \circ d_{P / T}: \mathcal{O}_{P} \stackrel{d_{P / T}}{\longrightarrow} \Omega_{P / T}^{1} \rightarrow \pi^{*} \Omega_{T}^{2},
$$

where $\mu$ is as in (4.3.2).

(iii) There is an induced map $\delta: M^{n} \rightarrow M^{n+1}$ making $M^{*}$ a differential graded algebra. The quotient map $\Omega_{P}^{*} \rightarrow \pi^{*} \Omega_{T}^{*} \rightarrow M^{*}$ is a map of differential graded $\mathcal{O}_{P}$-algebras.

Proof. We will give a somewhat different construction of $M^{*}$ which we will show coincides with that defined by (4.3.3).

Let $Y$ be a scheme, and let $\mathcal{F}$ be a vector bundle on $Y$. Let $\pi_{1}$ : $P_{1}:=\mathbb{P}(\mathcal{F}) \rightarrow Y$. Let $\mathcal{I} \subset \Omega_{Y}^{*}$ be a differential graded ideal, and write $M_{0}^{*}=\Omega_{Y}^{*} / \mathcal{I}$. (All our differential graded ideals will be trivial in degree 0 , so $M_{0}^{0}=\mathcal{O}_{Y}$.) Assume we are given an $M_{0}$-connection $\nabla: \mathcal{F} \rightarrow \mathcal{F} \otimes M_{0}^{1}$ in an obvious sense, that is a $k$ linear map fulfilling the "Leibniz" rule $\nabla(\lambda f)=\delta(\lambda) f+\lambda \nabla(f)$ for $\lambda \in \mathcal{O}_{Y}, f \in \mathcal{F}$. Define $\mathcal{J}:=\pi_{1}^{-1} \mathcal{I} \Omega_{P_{1}}^{*} \subset \Omega_{P_{1}}^{*}$, and let $\widetilde{M}^{*}:=\Omega_{P_{1}}^{*} / \mathcal{J}$. As a consequence of the Leibniz rule, the pullback $\pi_{1}^{*} \mathcal{F}$ has a $\widetilde{M}^{*}$-connection $\widetilde{\nabla}: \pi_{1}^{*} \mathcal{F} \rightarrow$ $\pi_{1}^{*} \mathcal{F} \otimes \widetilde{M^{1}}$.

We want to construct a quotient differential graded algebra $\widetilde{M}^{*} \rightarrow$ $M_{1}^{*}$ such that with respect to the quotient $M_{1}$-connection, the universal sequence

$$
0 \rightarrow \Omega_{P_{1} / Y}^{1}(1) \stackrel{j}{\longrightarrow} \pi_{1}^{*} \mathcal{F} \stackrel{q}{\longrightarrow} \mathcal{O}_{P_{1}}(1) \rightarrow 0
$$

is horizontal. The composition

$$
\Omega_{P_{1} / Y}^{1}(1) \stackrel{j}{\longrightarrow} \pi_{1}^{*} \mathcal{F} \stackrel{\tilde{\nabla}}{\longrightarrow} \pi_{1}^{*} \mathcal{F} \otimes \widetilde{M}^{1} \stackrel{q \otimes 1}{\longrightarrow} \widetilde{M}^{1}(1)
$$


is easily checked to be $\mathcal{O}_{P_{1}}$-linear. Let $\widetilde{\mathcal{K}_{1}} \subset \widetilde{M^{1}}$ denote the image of the above map twisted by $\mathcal{O}_{P_{1}}(-1)$. Define $\widetilde{\mathcal{K}^{*}} \subset \widetilde{M^{*}}$ to be the graded ideal generated by $\widetilde{\mathcal{K}^{1}}$ in degree 1 and $\delta \widetilde{\mathcal{K}^{1}}$ in degree 2 . Let $M_{1}^{*}:=\widetilde{\mathcal{M}^{*}} / \widetilde{\mathcal{K}^{*}}$. It is immediate that $M_{1}^{*}$ is a differential graded algebra, and that the subbundle $\Omega_{P_{1} / Y}^{1}(1) \subset \pi_{1}^{*} \mathcal{F}$ is horizontal for the quotient connection $\pi_{1}^{*} \mathcal{F} \rightarrow \pi_{1}^{*} \mathcal{F} \otimes M_{1}^{1}$.

Now let $P$ denote the flag bundle for $\mathcal{F}$. Realize $P$ as a tower of projective bundles

$$
P=P_{N-1} \rightarrow \ldots \rightarrow P_{2} \rightarrow P_{1} \rightarrow Y
$$

where $P_{i}$ is the projective bundle on the tautological subbundle on $P_{i-1}$. Starting with an $M_{0}$-connection on $\mathcal{F}$ on $Y$, we can iterate the above construction to get a sheaf of differential graded algebras $M_{i}^{*}$ on $P_{i}$, and an $M_{i}$-connection on $\mathcal{F} \mid P_{i}$ such that the tautological partial flag is horizontal. Let $M^{* *}$ be the resulting sheaf of differential graded algebras on $P$.

Suppose $M_{0}^{1}=\Omega_{Y}^{1}$. We will show by induction on $i$ that $M_{i}^{1} \cong$ $\Omega_{Y}^{1} \mid P_{i}$ in such a way that the surjection $\Omega_{P_{i}}^{1} \rightarrow M_{i}^{1}$ splits the natural inclusion $\Omega_{Y}^{1} \mid P_{i} \hookrightarrow \Omega_{P_{i}}^{1}$, or in other words that the kernel of the former is complementary to the image of the latter. This assertion is local on $P_{i-1}$ (in fact, it is local on $P_{i}$ ), so we may assume $P_{i}=\mathbb{P}(\mathcal{G})$ where $\mathcal{G}$ is trivial on $P_{i-1}$. We can then lift the $M_{i-1}$-connection on $\mathcal{G}$ to an $\Omega_{P_{i-1}}^{1}$-connection. The analog of (4.4.2) is now

$$
\Omega_{P_{i} / P_{i-1}}^{1}(1) \rightarrow \mathcal{G}\left|P_{i} \rightarrow \mathcal{G}\right| P_{i} \otimes \Omega_{P_{i}}^{1} \rightarrow \Omega_{P_{i}}^{1}(1)
$$

This composition twisted by $\mathcal{O}_{P_{i}}(-1)$ is shown in [7] (0.6.1) to be (upto sign) a splitting of $\Omega_{P_{i}}^{1} \rightarrow \Omega_{P_{i} / P_{i-1}}^{1}$. In particular, its image is complementary to $\Omega_{P_{i-1}}^{1} \mid P_{i}$. Factoring out $\Omega_{P_{i}}^{1}$ by the image of this map and by the pullback of the kernel of $\Omega_{P_{i-1}}^{1} \rightarrow M_{i-1}^{1} \cong \Omega_{Y}^{1} \mid P_{i-1}$, it follows easily that $M_{i}^{1} \cong \Omega_{Y}^{1} \mid P_{i}$ as claimed.

To show $M^{\prime *}$ as constructed here coincides with $M^{*}$ from (4.3.3) we must prove for $Y=T$ and $\mathcal{F}=\mathcal{E}$ that $M^{2} \cong M^{\prime 2}$. We filter $\Omega_{P / T}^{1}$ so fil $_{0}=(0)$ and $\operatorname{gr}_{i}=\Omega_{P_{i} / P_{i-1}}^{1} \mid P$. We will show by induction on $i$ that with reference to (4.3.3) we have

$$
\mu\left(\operatorname{gr}_{i} \Omega_{P / T}^{1}\right)=\delta\left(\mathcal{K}_{i}^{1}\right) \subset\left(\Omega_{T}^{2} \mid P\right) / \mu\left(\mathrm{fil}_{i-1} \Omega_{P / T}^{1}\right)
$$

where $\mathcal{K}_{i}^{1}$ is the image of $\Omega_{P_{i} / P_{i-1}}^{1} \mid P$ in $\Omega_{T}^{1} \mid P$ under the map analogous to (4.4.2). Suppose first $i=1$. Let $e_{0}, e_{1}, \ldots$ be a basis of $\mathcal{E}$, and let $t_{i}$ be the corresponding homogeneous coordinates on $P_{1}=\mathbb{P}(\mathcal{E})$ so 
ALGEBRAIC CHERN-SIMONS THEORY

$q\left(e_{i}\right)=t_{i}$ in (4.4.1). The inclusion $j: \Omega_{P_{1} / T}^{1}(1) \hookrightarrow \pi_{1}^{*} \mathcal{E}$ is given by

$$
t_{0} d\left(t_{i} / t_{0}\right) \mapsto e_{i}-\left(t_{i} / t_{0}\right) e_{0}
$$

Consider the diagram

$$
\begin{array}{ccccc}
\Omega_{P_{1} / T}^{1}(1) & \rightarrow & \mathcal{K}^{1}(1) \subset \Omega_{P_{1}}^{1}(1) & \stackrel{a}{\rightarrow} & \left(\Omega_{P_{1}}^{2} / \mathcal{K}^{1} \wedge \Omega^{1}\right)(1) \\
j \downarrow & & \uparrow & & \uparrow q \otimes 1 \\
\pi_{1}^{*} \mathcal{E} & \stackrel{\pi_{1}^{*} \Psi}{\longrightarrow} & \pi_{1}^{*} \mathcal{E} \otimes \Omega_{P_{1}}^{1} & \stackrel{\pi_{1}^{*} \Psi}{\longrightarrow} & \pi_{1}^{*} \mathcal{E} \otimes \Omega_{P_{1}}^{2}
\end{array}
$$

It is straightforward to check that $q \otimes 1 \circ \pi_{1}^{*} \Psi$ factorizes through $\Omega_{P_{1}}^{1}(1)$, thereby defining the dashed arrow $a$, and that for $\kappa \in \mathcal{K}^{1}$ we have $a\left(\kappa \otimes t_{i}\right)=d \kappa \otimes t_{i} \in\left(\Omega_{P_{1}}^{2} / \mathcal{K}^{1} \wedge \Omega^{1}\right)(1)$. Thus $M^{\prime 2}=\Omega_{P_{1}}^{2} /\left(\mathcal{K}^{1} \wedge \Omega^{1}+d \mathcal{K}^{1}\right)$ is obtained by factoring out on the upper right of (4.4.5) by the image of the composition across the top twisted by $\mathcal{O}_{P_{1}}(-1)$. Note that the composition across the bottom is the curvature $F(\Psi)$. If we write $\left(f_{i j}\right)$ for the curvature matrix with respect to the basis $e_{0}, e_{1}, \ldots$, we find using (4.4.4) that e.g. on the open set $t_{0} \neq 0, d \mathcal{K}^{1}$ is generated by elements

$$
\begin{gathered}
t_{0}^{-1}(q \otimes 1) F(\Psi)\left(e_{j}-\left(t_{j} / t_{0}\right) e_{0}\right)= \\
\sum_{i} f_{i j}\left(t_{i} / t_{0}\right)-\left(t_{j} / t_{0}\right) \sum_{k} f_{k 0}\left(t_{k} / t_{0}\right)
\end{gathered}
$$

On the other hand, the map $\iota$ in (4.3.2) is given by

$$
\begin{gathered}
\mathcal{O}_{P_{1}}(-1) \hookrightarrow \pi_{1}^{*} \mathcal{E}^{\vee} ; t_{i}^{-1} \mapsto \sum_{j}\left(t_{j} / t_{i}\right) e_{j}^{\vee} \\
\Omega_{P_{1} / T}^{1} \hookrightarrow \pi_{1}^{*} \mathcal{E}(-1) \hookrightarrow \pi_{1}^{*} \mathcal{E} \otimes \pi_{1}^{*} \mathcal{E}^{\vee} \\
d\left(t_{j} / t_{0}\right) \mapsto\left(e_{j}-\left(t_{j} / t_{0}\right) e_{0}\right) \otimes \sum_{i}\left(t_{i} / t_{0}\right) e_{i}^{\vee}
\end{gathered}
$$

The map $\mu$ from (4.3.2) is given by $\mu\left(e_{i}^{\vee} \otimes e_{j}\right)=f_{i j}$ hence by (4.4.7) we get

$$
\mu\left(d\left(t_{j} / t_{0}\right)\right)=\sum_{i}\left(t_{i} / t_{0}\right) f_{i j}-\sum_{i}\left(t_{i} t_{j} / t_{0}^{2}\right) f_{i 0} .
$$

Comparing (4.4.6) and (4.4.8), we conclude that (4.4.3) holds for $i=1$. The inductive step is precisely the same. We have $P_{i+1}=\mathbb{P}\left(\mathcal{G}_{i}\right)$ for some subbundle $\mathcal{G}_{i} \subset \mathcal{G}_{i-1} \mid P_{i}$. The question is local, so we may assume $\mathcal{G}_{i}$ is free. We assume inductively that $G_{i-1}$ has a $M_{i-1}=\Omega_{P_{i-1}}^{*} / \mathcal{I}_{i-1}^{*}$ connection. Define $\widetilde{M_{i-1}}=\Omega_{P_{i}}^{*} / \mathcal{I}_{i-1}^{*} \cdot \Omega_{P_{i}}^{*}$, so $\mathcal{G}_{i-1} \mid P_{i}$ has a $\widetilde{M}_{i-1^{-}}$ connection. One factors out by the image $\mathcal{K}_{i}^{1}$ of $\Omega_{P_{i} / P_{i-1}}^{1}$ as in (4.4.2) to define $M_{i}^{1}$ and the writes down a diagram like (4.4.5) to compare $d \mathcal{K}_{i}^{1}$ with the image of $\mu$ as in (4.3.2). At this point it is good to remark 
that the curvature $F_{\tau}: \mathcal{O}_{Q}(1) \rightarrow \pi^{*} \Omega_{T}^{1} \otimes \mathcal{O}_{Q}(1) \rightarrow M^{2} \otimes \mathcal{O}_{Q}(1)$ does not vanish. For example, for $N=2$, one has $F_{\tau}\left(t_{0}\right)=\left(f_{00}+f_{01}\left(t_{1} / t_{0}\right)\right) t_{0}$.

The remaining assertions in proposition 4.4.1 are easily verified.

Proposition 4.5.1. We have $\mathbb{R} \pi_{*} M^{i} \cong \pi_{*} M^{i}$ for $i<q$. The complex $H^{0}\left(T, \pi_{*} M^{*}\right)$ has no cohomology in odd degrees $<q$. For $2 n<q$, the map

$$
\delta: \mathbb{H}^{n-1}\left(P, M^{n} \rightarrow \ldots \rightarrow M^{2 n-1}\right) \rightarrow H^{0}\left(P, M^{2 n}\right)
$$

is injective.

4.6. We postpone the proof of proposition 4.5.1 for a while in order to finish the proof of theorem 4.0.1. Note first that since the curvature of the original bundle $E$ on $X$ is zero, the construction of proposition 4.4.1 above applied to $E$ and the flag bundle $Q$ yields a structure of differential graded algebra on $\pi^{*} \Omega_{X}^{*}$, and we have (from (4.1.1) a pullback map of complexes of sheaves on $P$

$$
\varphi^{*}: M^{*} \rightarrow R \varphi_{*} \pi^{*} \Omega_{X}^{*}
$$

coming from $\varphi^{*} M^{i} \rightarrow \pi^{*} \Omega_{X}^{i}$.

We will construct classes $\widetilde{c}$ and $\widetilde{w}$ in $\mathbb{H}^{n-1}\left(P, M^{n} \rightarrow \ldots \rightarrow M^{2 n-1}\right)$ such that with reference to the maps

$$
\begin{gathered}
\mathbb{H}^{n-1}\left(Q, \pi^{*} \Omega_{X}^{n} \rightarrow \ldots \rightarrow \pi^{*} \Omega_{X}^{2 n-1}\right) \underset{\alpha}{\downarrow} \stackrel{\gamma}{\cong} H^{0}\left(X, \Omega_{X}^{2 n-1}\right) / d H^{0}\left(X, \Omega_{X}^{2 n-2}\right) \\
\mathbb{H}^{n}\left(Q, \mathcal{K}_{n}^{m} \rightarrow \pi^{*} \Omega_{X}^{n} \rightarrow \ldots \rightarrow \pi^{*} \Omega_{X}^{2 n-1}\right) \\
\beta \uparrow \\
\mathbb{H}^{n}\left(Q, \mathcal{K}_{n}^{m} \rightarrow \pi^{*} \Omega_{X}^{\geq n}\right)
\end{gathered}
$$

we have

$$
\begin{aligned}
\beta \pi^{*}\left(c_{n}(E, \nabla)\right) & =\alpha \varphi^{*} \widetilde{c} \\
w_{n}(E, \nabla) & =\gamma^{-1} \varphi^{*} \widetilde{w}
\end{aligned}
$$

(Note that to avoid confusion between $H^{0}\left(\Omega_{X}^{2 n-1} / d \Omega_{X}^{2 n-2}\right)$ and $H^{0}\left(\Omega_{X}^{2 n-1}\right) / d H^{0}\left(\Omega_{X}^{2 n-2}\right)$, it is a good idea here to localize more and replace $X$ by its function field $\operatorname{Spec}(k(X))$. Note also that $\beta$ is always injective, and that $\gamma$ is an isomorphism because $X$ is affine).

We then show

$$
\delta \widetilde{c}=\delta \widetilde{w} \in H^{0}\left(P, M^{2 n}\right),
$$


whence, by proposition 4.5.1 we have $\widetilde{c}=\widetilde{w}$. Now consider the analogue of (4.6.2) down on $X$, with $\pi^{*} \Omega$ replaced by $\Omega$. Write $\alpha_{X}, \beta_{X}, \gamma_{X}$ for the corresponding maps. The assertion of theorem 4.0.1 is

$$
\gamma_{X}\left(w_{n}(E, \nabla)\right)=\beta_{X}\left(c_{n}(E, \nabla)\right) \text {. }
$$

It follows from (4.6.2) and evident functoriality of $\pi^{*}$ that (4.6.5) holds after pullback by $\pi^{*}$. Theorem 4.0.1 then follows from

Lemma 4.6.1. The pullback

$$
\begin{aligned}
\pi^{*}: \mathbb{H}^{n}\left(X, \mathcal{K}_{n}^{m} \rightarrow \Omega_{X}^{n} \rightarrow \ldots\right. & \left.\rightarrow \Omega_{X}^{2 n-1}\right) \\
& \rightarrow \\
& \mathbb{H}^{n}\left(Q, \mathcal{K}_{n}^{m} \rightarrow \pi^{*} \Omega_{X}^{n} \rightarrow \ldots \rightarrow \pi^{*} \Omega_{X}^{2 n-1}\right)
\end{aligned}
$$

is injective.

Proof of lemma. This is central to the splitting principle involved in the construction of characteristic classes in [8]. (See the argument on p. 52 in the proof of theorem 1.7 of (op. cit.).) An evident diagram chase involving cohomology of the $\mathcal{K}$-sheaves and the sheaves $\Omega$ and $\pi^{*} \Omega$ reduces one to showing the $\mathcal{K}$-cohomology groups $H^{n-1}\left(X, \mathcal{K}_{n}^{m}\right)$ and $H^{n-1}\left(Q, \mathcal{K}_{n}^{m}\right)$ have the same image in

$$
\mathbb{H}^{n-1}\left(Q, \pi^{*} \Omega_{X}^{n} \rightarrow \ldots \rightarrow \pi^{*} \Omega_{X}^{2 n-1}\right) .
$$

This follows because the multiplication map

$$
H^{n-2}\left(Q, \mathcal{K}_{n-1}^{m}\right) \otimes H^{1}\left(Q, \mathcal{K}_{1}\right) \rightarrow H^{n-1}\left(Q, \mathcal{K}_{n}^{m}\right) / \pi^{*} H^{n-1}\left(X, \mathcal{K}_{n}^{m}\right)
$$

is surjective. The line bundles on $Q$ have integrable $\tau$-connections in the sense of [8], so their classes in $\mathbb{H}^{1}\left(Q, \pi^{*} \Omega_{X}^{1} \rightarrow \ldots\right)$ vanish.

4.7. We turn now to the construction of the classes $\widetilde{c}$ and $\widetilde{w}$. One has

$$
\begin{aligned}
w\left(\mathcal{E}, \Psi, P_{n}\right) \in H^{0}\left(\Omega_{T}^{2 n-1}\right) / d H^{0}\left(\Omega_{T}^{2 n-2}\right) & \\
& \cong \mathbb{H}^{n-1}\left(T, \Omega_{T}^{n} \rightarrow \ldots \rightarrow \Omega_{T}^{2 n-1}\right)
\end{aligned}
$$

We define $\widetilde{w}$ by the natural pullback

$$
\widetilde{w}=\pi^{*} w\left(\mathcal{E}, \Psi, P_{n}\right) \in \mathbb{H}^{n-1}\left(P, M^{n} \rightarrow \ldots \rightarrow M^{2 n-1}\right) .
$$

It follows that

$$
\delta(\widetilde{w})=\pi^{*}\left(d w\left(\mathcal{E}, \Psi, P_{n}\right)\right)=\pi^{*}\left(P_{n}(F(\Psi))\right) \in H^{0}\left(P, M^{2 n}\right) .
$$

To construct $\widetilde{c}$, we remark first that the map

$$
\mathbb{H}^{n-1}\left(P, M^{n} \rightarrow \ldots \rightarrow M^{2 n-1}\right) \rightarrow \mathbb{H}^{n}\left(P, \mathcal{K}_{n}^{m} \rightarrow M^{n} \rightarrow \ldots \rightarrow M^{2 n-1}\right)
$$

is injective, so it suffices to construct

$$
\widetilde{c} \in \operatorname{ker}\left(\mathbb{H}^{n}\left(P, \mathcal{K}_{n}^{m} \rightarrow M^{n} \rightarrow \ldots \rightarrow M^{2 n-1}\right) \rightarrow H^{n}\left(P, \mathcal{K}_{n}^{m}\right)\right)
$$


This injectivity follows easily from the structure of $H^{n-1}\left(P, \mathcal{K}_{n}^{m}\right)$, given that $P$ is a flag bundle over affine space, but in fact the construction of $\widetilde{c}$ as in (4.7.3) would suffice for our purposes anyway, so we won't give the argument in detail.

Let $\ell_{i}$ be the rank one subquotients of $\pi^{*} \mathcal{E}$. A basic result from [7] is that $\pi^{*} \mathcal{E}$ admits a "connection" with values in $M^{*}$,

$$
\pi^{*} \mathcal{E} \rightarrow \pi^{*} \mathcal{E} \otimes_{\mathcal{O}_{P}} M^{1}
$$

and that the filtration defining the $\ell_{i}$ is horizontal for this "connection". Thus there exist local transition functions $f_{\alpha, \beta}^{i}$ and local connection forms $\omega_{\alpha}^{i} \in M^{1}$ verifying

$$
d \log f^{i}=\partial \omega^{i},
$$

and thus defining $\ell_{i} \in \mathbb{H}^{1}\left(P, \mathcal{K}_{1} \rightarrow M^{1}\right)$. Here $\partial$ is the Cech differential. Then $\widetilde{c}$ is defined by the cocyle

$$
\left(x^{\prime}, x^{n}, \ldots, x^{2 n-1}\right) \in\left(\mathcal{C}^{n}\left(\mathcal{K}_{n}\right) \times \mathcal{C}^{n-1}\left(M^{n}\right) \ldots \times \mathcal{C}^{0}\left(M^{2 n-1}\right)\right)_{d-\partial}
$$

with

$$
\begin{gathered}
x^{\prime}=\sum_{i_{1}<\ldots<i_{n}} f^{i_{1}} \cup \ldots \cup f^{i_{n}} \\
x^{n}=\sum_{i_{1}<\ldots<i_{n}} \omega^{i_{1}} \wedge \partial \omega^{i_{2}} \wedge \ldots \wedge \partial \omega^{i_{n}} \\
x^{n+1}=\sum_{i_{1}<\ldots<i_{n}} \delta \omega^{i_{1}} \wedge \omega^{i_{2}} \wedge \partial \omega^{i_{3}} \wedge \ldots \wedge \partial \omega^{i_{n}} \\
x^{2 n-1}=\sum_{i_{1}<\ldots<i_{n}} \delta \omega^{i_{1}} \wedge \ldots \wedge \delta \omega^{i_{n-1}} \wedge \omega^{i_{n}} .
\end{gathered}
$$

The cup products " $\cup$ " here are Cech products. By definition [8], $\beta \pi^{*}\left(c_{n}(E, \nabla)=\varphi^{*} \widetilde{c}\right.$. Applying $\delta$ to the last equation, it follows that the image of $\widetilde{c}$ in $H^{0}\left(P, M^{2 n}\right)$ is

$$
\sum_{i_{1}<\ldots<i_{n}} F\left(\ell_{i_{1}}\right) \wedge \ldots \wedge F\left(\ell_{i_{n}}\right)
$$

This is exactly $P_{n}\left(F\left(\oplus \ell_{i}\right)\right)=\pi^{*} P_{n}(F(\Psi))$. (As $M^{*}$ is a quotient complex of $\Omega_{P}^{*}$ by 4.4.1, (iii), invariance for $P_{n}$ guarantees independence of the choice of local bases for $\pi^{*} \mathcal{E}$.) Comparing this with (4.7.2), we conclude $\delta \widetilde{c}=\delta \widetilde{w}$ so (4.6.4) holds. 
4.8. We turn now to proof of proposition 4.5.1.

Proposition 4.8.1. The Koszul complex associated to (4.3.3

$$
\begin{gathered}
\ldots \rightarrow \Omega_{P / T}^{2} \otimes_{\mathcal{O}_{P}} \pi^{*} \Omega_{T}^{*}[-4] \rightarrow \Omega_{P / T}^{1} \otimes_{\mathcal{O}_{P}} \pi^{*} \Omega_{T}^{*}[-2] \stackrel{\mu \otimes 1}{\longrightarrow} \\
\pi^{*} \Omega_{T}^{*} \rightarrow M^{*} \rightarrow 0
\end{gathered}
$$

is exact in degrees $<q$.

To clarify and simplify the argument, we will use commutative algebra. Let $B$ be a commutative ring. Let $C$ be a commutative, graded $B$-algebra, and let $S$ be a graded $C$-module. Let $Z$ be a finitely generated free $B$-module with generators $\epsilon_{\alpha}$, and let $\nu: Z \rightarrow C_{2}$, with $\nu\left(\epsilon_{\alpha}\right)=f_{\alpha}$. Let $\mathcal{I} \subset C$ be the ideal generated by the $f_{\alpha}$. Write $\operatorname{gr}_{\mathcal{I}}(S):=\oplus \mathcal{I}^{n} S / \mathcal{I}^{n+1} S$. Note $\operatorname{gr}_{\mathcal{I}}(S)$ is a graded module for the symmetric algebra $B[Z]$ (with $Z$ in degree 2). The dictionary we have in mind is

$$
\begin{aligned}
B & =\Gamma\left(T, \mathcal{O}_{T}\right) \\
C & =\Omega_{T}^{\text {even }} \subset S=\Omega_{T}^{*} \\
Z & =H o m(\mathcal{E}, \mathcal{E}) \\
f_{\alpha}=f_{i j} & =\text { entries of curvature matrix }
\end{aligned}
$$

Lemma 4.8.2. Let $d \geq 2$ be given. The following are equivalent.

(i) The evident map

$$
\rho:(S / \mathcal{I} S)[Z]:=(S / \mathcal{I} S) \otimes_{B} B[Z] \rightarrow g r_{\mathcal{I}}(S)
$$

is an isomorphism in degrees $\leq d$.

(ii) For all $\alpha$, the multiplication map

$$
f_{\alpha}: S /\left(f_{1}, \ldots, f_{\alpha-1}\right) S \rightarrow S /\left(f_{1}, \ldots, f_{\alpha-1}\right) S
$$

is injective in degrees $\leq d$.

Proof. This amounts to redoing the argument in Chapter 0, §(15.1.1)(15.1.9) of [11] in a graded situation, where the hypotheses and conclusions are asserted to hold only in degrees $\leq d$. The argument may be sketched as follows.

Step 1. Suppose $\alpha=1$, and write $f=f_{1}$. Let $\operatorname{gr}(S)=\oplus f^{n} S / f^{n+1} S$. Suppose the kernel of multiplication by $f$ on $S$ is contained in degrees $>d$. Then the natural map $\varphi:(S / f S)[T] \rightarrow \operatorname{gr}(S)$ is an isomorphism in degrees $\leq d$. Here, of course, $T$ is given degree $=\operatorname{degree}(f)=2$.

Indeed, $\varphi$ is always surjective, and injectivity in degrees $\leq d$ amounts to the assertion that for $x \in S$ of degree $\leq d-2 k$ with $f^{k} x=f^{k+1} y$, we have $x=f y$. This is clear. 
Step 2. Suppose now the condition in (ii) holds. We prove (i) by induction on $\alpha$. We may assume by step 1 that $\alpha>1$. Let $\mathcal{J}$ (resp. $\mathcal{I})$ be the ideal generated by $f_{1}, \ldots, f_{\alpha-1}$ (resp. $\left.f_{1}, \ldots, f_{\alpha}\right)$. Write $\operatorname{gr}_{\mathcal{J}}(S)=\oplus J^{n} S / J^{n+1} S$. By induction, we may assume

$$
S / \mathcal{J} S\left[T_{1}, \ldots, T_{\alpha-1}\right] \rightarrow \operatorname{gr}_{\mathcal{J}}(S)
$$

is an isomorphism in degrees $\leq d$. We have to show the same for

$$
\psi: \operatorname{gr}_{\mathcal{J}}(S) / f_{\alpha} \operatorname{gr}_{\mathcal{J}}(S)\left[T_{\alpha}\right] \rightarrow \operatorname{gr}_{\mathcal{I}}(S) .
$$

By (4.8.4) we have that multiplication by $f_{\alpha}$ on $\operatorname{gr}_{\mathcal{J}}(S)$ is injective in degrees $\leq d$ (where the degree grading comes from $S$, not the $\operatorname{gr}_{\mathcal{J}}$ grading). An easy argument shows the multiplication map

$$
f_{\alpha}: S / \mathcal{J}^{r} S \hookrightarrow S / \mathcal{J}^{r} S
$$

is injective in degrees $\leq d$ for all $r$. Define

$$
\begin{gathered}
\left.\left(Q_{k}\right)_{i}=\sum_{j \leq k-i} \operatorname{gr}_{\mathcal{J}}^{k-j}(S) / f_{\alpha} \operatorname{gr}_{\mathcal{J}}^{k-j}(S)\right) T^{j} \\
\left(Q_{k}\right)_{0}=Q_{k} \quad\left(Q_{k}\right)_{k+1}=(0) \\
\operatorname{gr}^{i}\left(Q_{k}\right)=\left(\operatorname{gr}_{\mathcal{J}}^{k-i}(S) / f_{\left.\alpha r_{\mathcal{J}}^{k-i}(S)\right) T^{i}}\right.
\end{gathered}
$$

Define

$$
Q_{k}^{\prime}=\psi\left(Q_{k}\right) \quad\left(Q_{k}^{\prime}\right)_{i}=\psi\left(\left(Q_{k}\right)_{i}\right) \quad \operatorname{gr}^{i}\left(Q_{k}^{\prime}\right)=\left(Q_{k}^{\prime}\right)_{i} /\left(Q_{k}^{\prime}\right)_{i+1} .
$$

The map $\psi$ is surjective, so it will suffice to show the maps

$$
\operatorname{gr}^{i}\left(Q_{k}\right) \rightarrow \operatorname{gr}^{i}\left(Q_{k}^{\prime}\right)
$$

are injective in $(S)$-degrees $\leq d$. The left hand side is

$$
\mathcal{J}^{i} S /\left(f_{\alpha} \mathcal{J}^{i} S+\mathcal{J}^{i+1} S\right) T^{k-i}
$$

The right hand side of (4.8.8) is the image of

$$
\mathcal{J}^{k} S+f_{\alpha} \mathcal{J}^{k-1} S+\ldots+f_{\alpha}^{k-i-1} \mathcal{J}^{i+1} S
$$

in $\mathcal{I}^{k} S / \mathcal{I}^{k+1} S$. What we have to show is that for $x \in \mathcal{J}^{i} S$ of degree $\leq d-2(k-i)$, the inclusion

$$
f_{\alpha}^{k-i} x \in \mathcal{J}^{k} S+f_{\alpha} \mathcal{J}^{k-1}+\ldots+f_{\alpha}^{k-i-1} \mathcal{J}^{i+1}+\mathcal{I}^{k+1} S
$$

implies $x \in f_{\alpha} \mathcal{J}^{i} S+\mathcal{J}^{i+1} S$. The right side of (4.8.9) is contained in $\mathcal{J}^{i+1} S+\mathcal{I}^{k+1} S \subset \mathcal{J}^{i+1} S+f_{\alpha}^{k+1-i} S$. Multiplication by $f_{\alpha}$ on $S / \mathcal{J}^{i+1} S$ is injective in degrees $\leq d$ by (4.8.6), so $f_{\alpha}^{k-i} x \in f_{\alpha}^{k+1-i} S+\mathcal{J}^{i+1} S$ implies there exists $y \in S$ such that $x-f_{\alpha} y \in \mathcal{J}^{i+1} S$. Since $x \in \mathcal{J}^{i} S$, we have $f_{\alpha} y \in \mathcal{J}^{i} S$ whence by (4.8.6) again, $y \in \mathcal{J}^{i} S$ so $x \in f_{\alpha} \mathcal{J}^{i} S+\mathcal{J}^{i+1} S$. This completes the verification of step 2 . 
Step 3. It remains to show $(i) \Rightarrow(i i)$. Again we argue by induction on $\alpha$. Suppose first $\alpha=1$. Given $x \in S$ non-zero of degree $\leq d$ such that $f_{1} x=0$, it would follow from (i) that $x \in f_{1}^{N} S$ for all $N$, which is ridiculous by reason of degree. Now suppose $\alpha \geq 2$ and that (i) implies (ii) for $\alpha-1$. By assumption the map

$$
S / \mathcal{I} S\left[T_{1}, \ldots, T_{\alpha}\right] \rightarrow \operatorname{gr}_{\mathcal{I}}(S)
$$

is an isomorphism in degrees $\leq d$. In particular, multiplication by $f_{1}$ is injective in degrees $\leq d$ on $\operatorname{gr}_{\mathcal{I}} S$. Arguing as above, an $x \in S$ of degree $\leq d$ such that $f_{1} x=0$ would lie in $\mathcal{I}^{N} S$ for all $N$, a contradiction. Thus the first step in (ii) holds. To finish the argument, we may factor out by $f_{1}$, writing $\bar{S}=S / f_{1} S$. Let $\mathcal{K}$ be the ideal generated by $f_{2}, \ldots, f_{\alpha}$. Factoring out by $T_{1}$ on both sides of (4.8.10) yields

$$
\bar{S} / \mathcal{K} \bar{S}\left[T_{2}, \ldots, T_{\alpha}\right] \rightarrow \operatorname{gr}_{\mathcal{K}}(\bar{S})
$$

injective in degrees $\leq d$. We conclude by induction that (ii) holds for $\bar{S}$.

Continuing the dictionary from (4.8.2) above, the ring $R$ and the module $W$ in the lemma below correspond to the ring of functions on some affine in $P$ and the module of 1 -forms $\Omega_{P / T}^{1} \subset \pi^{*} \operatorname{Hom}(\mathcal{E}, \mathcal{E})$.

Lemma 4.8.3. Let notation be as above, and assume $\nu: Z \rightarrow C_{2}$ satisfies the equivalent conditions of lemma 4.8.9. Let $R$ be a flat $B$ algebra, and let $W \subset Z \otimes_{B} R$ be a free, split $R$-submodule with basis $g_{\beta}$. Then the multiplication maps

$$
g_{\beta}: S \otimes_{B} R /\left(g_{1}, \ldots, g_{\beta-1}\right) S \otimes_{B} R \rightarrow S \otimes_{B} R /\left(g_{1}, \ldots, g_{\beta-1}\right) S \otimes_{B} R
$$

are injective in degrees $\leq d$.

Proof. Assume not. We can localize at some prime of $R$ contained in the support for some element in the kernel of multiplication by $g_{\beta}$ and reduce to the case $R$ local. Then we may extend $\left\{g_{\beta}\right\}$ to a basis of $Z \otimes_{B} R$ and use the implication (i) $\Longrightarrow$ (ii) from lemma 4.8.2. Note that $\nu \otimes 1: Z \otimes R \rightarrow C_{2} \otimes R$ satisfies (i) by flatness.

Lemma 4.8.4. With notations as above, assume $Z$ satisfies the conditions of lemma 4.8 .9 for some $d \geq 2$. Let $J \subset R \otimes_{B} C$ be the ideal generated by $(1 \otimes \nu)(W)$. Then the Koszul complex

$$
\begin{aligned}
\ldots \rightarrow \wedge^{2} W \otimes_{R}\left(R \otimes_{B} S\right) \rightarrow W \otimes_{R}\left(R \otimes_{B} S\right) \rightarrow \\
R \otimes_{B} S \rightarrow\left(R \otimes_{B} S\right) / J \rightarrow 0
\end{aligned}
$$

is exact in degrees $\leq d$. 
Proof. To simplify notation, let $A=R \otimes_{B} C, M=R \otimes_{B} S, V=$ $W \otimes_{R} C$, so the Koszul complex becomes

$$
\ldots \wedge^{2} V \otimes_{A} M \rightarrow V \otimes_{A} M \rightarrow M \rightarrow M / J M \rightarrow 0 .
$$

We argue by induction on the rank of $V$. If this rank is 1 , the assertion is that the sequence

$$
0 \rightarrow M \stackrel{g_{1}}{\rightarrow} M \rightarrow M / g_{1} M \rightarrow 0
$$

is exact in degrees $\leq d$, which follows from lemma 4.8.3. In general, if $V$ has an $A$-basis $g_{1}, \ldots, g_{\beta}$, let $V^{\prime}$ be the span of $g_{1}, \ldots, g_{\beta-1}$. By induction, the Koszul complex

$$
\ldots \wedge^{2} V^{\prime} \otimes M \rightarrow V^{\prime} \otimes M \rightarrow M
$$

is a resolution of $M /\left(g_{1}, \ldots, g_{\beta-1}\right) M$ in degrees $\leq d$. If we tensor this module with the two-term complex $A \stackrel{g_{\beta}}{\longrightarrow} A$ we obtain a complex which by lemma 4.8 .3 is quasi-isomorphic to $M /\left(g_{1}, \ldots, g_{\beta}\right) M$ in degrees $\leq d$. On the other hand, this complex is quasi-isomorphic to the complex obtained by tensoring $A \stackrel{g_{\beta}}{\longrightarrow} A$ with the above $V^{\prime}$-Koszul complex, and this tensor product is identified with the $V$-Koszul complex.

For our application, $B=\mathbb{C}\left[x_{i j}^{(k)}, y_{i j}^{(k)}\right]$ is the polynomial ring in two sets of variables, with $1 \leq i, j \leq N=\operatorname{dim}(E)$ and $1 \leq k \leq q$ for some large integer $q$. Let $\Omega$ be the free $B$-module on symbols $d x_{i j}^{(k)}$ and $d y_{i j}^{(k)}$. Let $S=\bigwedge_{B} \Omega$, graded in the obvious way with $d x$ and $d y$ having degree 1 , and let $C=S_{\text {even }}$ be the elements of even degree. Define

$$
a_{i j}=\sum_{\ell=1}^{q} x_{i j}^{(\ell)} d y_{i j}^{(\ell)} ; \quad f_{i j}=d a_{i j}-\sum_{m=1}^{N} a_{i m} \wedge a_{m j} .
$$

We have

$$
f_{i j}=\sum_{\ell=1}^{q} d x_{i j}^{(\ell)} d y_{i j}^{(\ell)}-\sum_{m, \ell, p} x_{i m}^{(\ell)} x_{m j}^{(p)} d y_{i m}^{(\ell)} \wedge d y_{m j}^{(p)} .
$$

Now give $S$ and $C$ a second grading according to the number of $d x$ 's in a monomial. We denote this grading by $z=\sum z(j)$. For example, $f_{i j}=f_{i j}(1)+f_{i j}(0)$ with $f_{i j}(1)=\sum_{k} d x_{i j}^{(k)} \wedge d y_{i j}^{(k)}$. Let $Z$ be the free $B$-module on symbols $\epsilon_{i j}$, with $1 \leq i, j \leq N$. We consider maps

$$
\mu, \mu(1): Z \rightarrow C_{2} ; \mu\left(\epsilon_{i j}\right)=f_{i j} ; \mu(1)\left(\epsilon_{i j}\right)=f_{i j}(1)
$$

Lemma 4.8.5. Suppose the map $\mu(1)$ above satisfies the conditions of lemma 4.8.9 above for some $d \geq 2$. Then so does $\mu$. 
Proof. Suppose

$$
f_{\alpha} \ell_{\alpha}=\sum_{1 \leq \beta \leq \alpha-1} f_{\beta} \ell_{\beta}
$$

with the $\ell_{\beta}$ homogeneous of some degree $<d$. Write

$$
\ell_{\beta}=\sum_{0 \leq j \leq r} \ell_{\beta}(j) ; \quad 1 \leq \beta \leq \alpha
$$

such that $\ell_{\beta}(r) \neq 0$ for some $\beta$. We have

$$
f_{\alpha}(1) \ell_{\alpha}(r)=\sum_{1 \leq \beta \leq \alpha-1} f_{\beta}(1) \ell_{\beta}(r)
$$

We want to show $\ell_{\alpha}$ belongs to the submodule generated by $f_{1}, \ldots, f_{\alpha-1}$, and we will argue by double induction on $r$ and on the set

$$
\mathcal{A}=\left\{\beta \leq \alpha \mid \ell_{\beta}(r) \neq 0\right\} .
$$

If $r=0$ and $\ell_{\alpha} \neq 0$, we get a contradiction from (5.3.1), since we have assumed the $\ell_{\beta}$ have degree $<d$, and $\ell_{\alpha}(0)$ cannot lie in the ideal generated by the $f_{\beta}(1)$. Assume now $r \geq 1$.

Case 1.- Suppose $\ell_{\alpha}(r) \neq 0$. From the above, we conclude we can write

$$
\ell_{\alpha}(r)=\sum_{\beta \in \mathcal{A}, \beta \neq \alpha} m_{\beta}(r-1) f_{\beta}(1)
$$

Define

$$
\begin{aligned}
& \ell_{\alpha}^{\prime}=\ell_{\alpha}-\sum_{\beta \in \mathcal{A}, \beta \neq \alpha} m_{\beta}(r-1) f_{\beta} \\
& \ell_{\beta}^{\prime}=\ell_{\beta}-m_{\beta}(r-1) f_{\alpha} ; \quad \beta \in \mathcal{A}, \beta \neq \alpha .
\end{aligned}
$$

We have still, taking $\ell_{\beta}^{\prime}=\ell_{\beta}$ for $\beta \notin \mathcal{A}$

$$
f_{\alpha} \ell_{\alpha}^{\prime}=\sum_{1 \leq \beta \leq \alpha-1} f_{\beta} \ell_{\beta}^{\prime}
$$

Since $\ell_{\beta}^{\prime}(r)=\ell_{\beta}(r)=0$ for $\beta \notin \mathcal{A}, \ell_{\alpha}^{\prime}(r)=0$, and $\ell_{\beta}^{\prime}(s)=0$ for $s>r$ and all $\beta$; the inductive hypothesis says $\ell_{\alpha}^{\prime}$ lies in the ideal generated by the $f_{\beta}$ for $\beta<\alpha$. It follows from (4.3.2) that $\ell_{\alpha}$ lies in this ideal also.

Case 2.- $\ell_{\alpha}(r)=0$. Choose $\gamma \in \mathcal{A}$. We have

$$
\sum_{\beta \in \mathcal{A}} f_{\beta}(1) \ell_{\beta}(r)=0
$$


Since the $f_{\beta}(1)$ are assumed to satisfy the equivalent hypotheses of lemma 4.6.1, we can write

$$
\ell_{\gamma}(r)=\sum_{\beta \in \mathcal{A}, \beta \neq \gamma} m_{\beta}(r-1) f_{\beta}(1) .
$$

As in (4.3.2), we write

$$
\begin{aligned}
& \ell_{\gamma}^{\prime}=\ell_{\gamma}-\sum_{\beta \in \mathcal{A}, \beta \neq \gamma} m_{\beta}(r-1) f_{\beta} \\
& \ell_{\beta}^{\prime}=\ell_{\beta}+m_{\beta}(r-1) f_{\gamma} ; \quad \beta \in \mathcal{A}, \beta \neq \gamma .
\end{aligned}
$$

Again, taking $\ell_{\beta}^{\prime}=\ell_{\beta}$ for $\beta \notin \mathcal{A}$, we get (4.3.3), so we may conclude by induction.

Lemma 4.8.6. The map $\mu(1)$ defined by

$$
\mu(1)\left(\epsilon_{i j}\right)=f_{i j}(1)=\sum_{\ell=1}^{q} d x_{i j}^{(\ell)} \wedge d y_{i j}^{(\ell)}
$$

satisfies the hypotheses of lemma 4.6.1 with $d=q-1$.

Proof. Let $V_{i j}$ be the $\mathbb{C}$-vector space of dimension $2 q$ with basis the $d x_{i j}^{(\ell)}$ and the $d y_{i j}^{(\ell)}$. Write $V=\oplus V_{i j}$. We have

$$
S=\bigwedge V \otimes B \cong \otimes_{i, j}\left(\bigwedge V_{i j}\right) \otimes B
$$

It is convenient to well-order the pairs $i j$, writing $f_{\alpha}(1)=f_{i j}(1) \in \bigwedge V_{\alpha}$. We have

$$
\begin{aligned}
& S /\left(f_{1}, \ldots, f_{\alpha-1}\right) S \cong \\
& \bigotimes_{\beta \geq \alpha}\left(\bigwedge V_{\beta}\right) \otimes \bigotimes_{\beta<\alpha}\left(\left(\bigwedge V_{\beta}\right) /\left(f_{\beta}(1) \bigwedge V_{\beta}\right)\right) \otimes B .
\end{aligned}
$$

It is clear from this that multiplication by $f_{\alpha}(1)$ will be injective in a given degree $d$ if the multiplication map $f_{\alpha}(1): \bigwedge V_{\alpha} \rightarrow \bigwedge V_{\alpha}$ is injective in degrees $\leq d$. It is clear from the shape of $f_{\alpha}(1)$ in (4.6.2) that multiplication by $f_{\alpha}(1)$ will be injective in degrees $\leq q-1$.

This completes the proof of proposition 4.8.1 above.

Proposition 4.9.1. We have for $i<q$

$$
\pi_{*} M^{0}=\mathcal{O}_{T} ; \quad \pi_{*} M^{1}=\Omega_{T}^{1} ; \quad R^{j} \pi_{*} M^{i}=(0) ; j \geq 1
$$

The sheaf $\pi_{*} M^{i}$ admits an increasing filtration $f i l_{\ell}\left(\pi_{*} M^{i}\right), \ell \geq 0$ which is stable under $\delta$ and satisfies

$$
g r_{j}\left(\pi_{*} M^{i}\right) \cong H^{j}\left(P, \Omega_{P / T}^{j}\right) \otimes \Omega_{T}^{i-2 j} \cong C H^{j}(P) \otimes_{\mathbb{Z}} \Omega_{T}^{i-2 j}
$$


for $j \geq 0$. Here $C H^{j}(P)$ is the Chow group of codimension $j$ algebraic cycles on $P$. The differential $g r_{j}\left(\pi_{*} M^{i}\right) \rightarrow g r_{j}\left(\pi_{*} M^{i+1}\right)$ is the identity on the Chow group tensored with the exterior derivative on $\Omega_{T}^{*}$ up to sign.

Note that the last assertion in (4.9.1) implies for $i<q$

$$
H^{*}\left(P, M^{i}\right) \cong \begin{cases}H^{0}\left(T, \pi_{*} M^{i}\right) & *=0 \\ 0 & * \geq 1 .\end{cases}
$$

It follows from (4.9.2) that the complex $H^{0}\left(T, M^{*}\right)$ has no cohomology in odd degrees $<q-1$. (Recall that $T$ has no higher de Rham cohomology). These assertions imply proposition 4.5.1.

Proof of proposition 4.9.1. The first two assertions in (4.9.1) are clear, because $M^{0}=\mathcal{O}_{P}$ and $M^{1}=\pi^{*} \Omega_{T}^{1}$. We define

$$
G_{j}=\operatorname{Im} \Omega_{P / T}^{j} \otimes \pi^{*} \Omega_{T}^{i-2 j} \rightarrow \Omega_{P / T}^{j-1} \otimes \pi^{*} \Omega_{T}^{i-2 j+2}, G_{0}=M^{i}
$$

coming from the resolution of $M^{i}$ in 4.8.1. Then $R^{a} \pi_{*} G_{j}=0$ for $a \neq j$. This proves $R^{j} \pi_{*} M^{i}=0$ for $j \geq 1$. One has a short exact sequence

$$
0 \rightarrow R^{j} \pi_{*} \Omega_{P / T}^{j} \otimes \Omega_{T}^{i-2 j} \rightarrow R^{j} \pi_{*} G_{j} \rightarrow R^{j+1} \pi_{*} G_{j+1} \rightarrow 0
$$

with $R^{0} \pi_{*} G_{0}=\pi_{*} M^{i}$. One defines

$$
\begin{aligned}
\operatorname{fil}_{j}\left(\pi_{*} M^{i}\right)=\text { inverse image of } R^{j} \pi_{*} \Omega_{P / T}^{j} \otimes & \Omega_{T}^{i-2 j} \\
& \text { via } R^{0} \pi_{*} G_{0} \rightarrow R^{j} \pi_{*} G_{j} .
\end{aligned}
$$

This proves (4.9.2).

In order to understand the map $\operatorname{gr}_{j}\left(\pi_{*} M^{i}\right) \rightarrow \operatorname{gr}_{j}\left(\pi_{*} M^{i+1}\right)$, we construct a commutative diagram

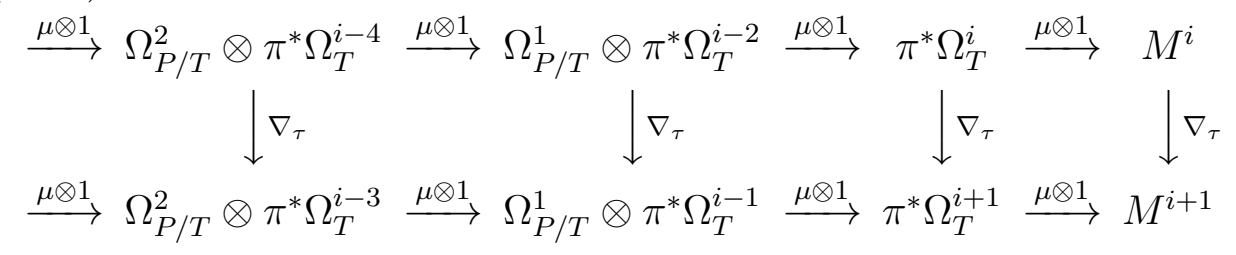

mapping the resolution of $M^{i}$ to the resolution of $M^{i+1}$ given by 4.8.1. To this aim recall that one has an exact sequence of complexes

$$
0 \rightarrow K^{*} \rightarrow \Omega_{P}^{*} \rightarrow M^{*} \rightarrow 0
$$


with

$$
K^{i}=\Omega_{P / T}^{i} \oplus \Omega_{P / T}^{i-1} \otimes \pi^{*} \Omega_{T}^{1} \ldots \oplus \Omega_{P / T}^{1} \otimes \pi^{*} \Omega_{T}^{i-1} \oplus \mu\left(\Omega_{P / T}^{1}\right) \wedge \pi^{*} \Omega_{T}^{i-2}
$$

Note that the differential $K^{i-j-1} \rightarrow K^{i-j}$ acts as follows

$$
\begin{gathered}
\Omega_{P / T}^{j} \otimes \pi^{*} \Omega_{T}^{i-1-2 j} \rightarrow \\
\Omega_{P / T}^{j+1} \otimes \pi^{*} \Omega_{T}^{i-1-2 j} \oplus \Omega_{P / T}^{j} \otimes \pi^{*} \Omega_{T}^{i-2 j} \oplus \Omega_{P / T}^{j-1} \otimes \pi^{*} \Omega_{T}^{i-2 j+1} .
\end{gathered}
$$

To see this, write

$$
\Omega_{P / T}^{j} \otimes \pi^{*} \Omega_{T}^{i-1-2 j}=\Omega_{P / T}^{j} \otimes_{\mathcal{O}_{T}} \pi^{-1} \Omega_{T}^{i-1-2 j}
$$

and apply the Leibniz rule with

$$
d \Omega_{P / T}^{1} \subset \Omega_{P / T}^{2} \oplus \Omega_{P / T}^{1} \otimes \pi^{*} \Omega_{T}^{1} \oplus \mu\left(\Omega_{P / T}^{1}\right) .
$$

The corresponding map $\Omega_{P / T}^{1} \rightarrow \mu\left(\Omega_{P / T}^{1}\right)$ is of course $\mu$. We denote by $\nabla_{\tau}$ the corresponding map $\Omega_{P / T}^{1} \rightarrow \Omega_{P / T}^{1} \otimes \pi^{*} \Omega_{T}^{1}$ and also by $\nabla_{\tau}$ the induced map $\Omega_{P / T}^{j} \otimes \pi^{*} \Omega_{T}^{i-1-2 j} \rightarrow \Omega_{P / T}^{j} \otimes \pi^{*} \Omega_{T}^{i-2 j}$. For $\gamma \in \Omega_{P / T}^{j} \otimes$ $\pi^{*} \Omega_{T}^{i-1-2 j}$, write $d \gamma=\gamma_{j+1}+\nabla_{\tau}(\gamma)+(\mu \otimes 1)(\gamma)$ with $\gamma_{j+1} \in \Omega_{P / T}^{j+1} \otimes$ $\pi^{*} \Omega_{T}^{i-1-2 j}$. The integrability condition $d^{2}(\gamma)=0$ in $\Omega_{P}^{*}$ says that $(\mu \otimes$ 1) $\nabla_{\tau}(\gamma)=\nabla_{\tau}(\mu \otimes 1)(\gamma) \in \Omega_{P / T}^{j-1} \otimes \pi^{*} \Omega_{T}^{i-j+2}$, upto sign.

Thus $\operatorname{gr}_{j} \pi_{*} M^{i} \rightarrow \operatorname{gr}_{j} \pi_{*} M^{i+1}$ is the map

$$
R^{j} \pi_{*} \nabla_{\tau}: R^{j} \pi_{*} \Omega_{P / T}^{j} \otimes \Omega_{T}^{i-2 j} \rightarrow R^{j} \pi_{*} \Omega_{P / T}^{j} \otimes \Omega_{T}^{i-2 j+1} .
$$

Now, $\nabla_{\tau}=d \mid K^{*}$, where $d$ is the differential of $\Omega_{P}^{*}$. Let $\ell_{i}$ be the rank one subquotients of $\pi^{*} \mathcal{E}$, with local algebraic transition functions $f_{\alpha, \beta}^{i}$. Then $R^{j} \pi_{*} \Omega_{P / T}^{j} \otimes \Omega_{T}^{i-2 j}$ is generated over $\mathcal{O}_{T}$ by elements $\varphi=F \wedge \omega$, with

$$
F=d \log f_{\alpha_{0}, \alpha_{1}}^{i_{1}} \wedge \cdots \wedge d \log f_{\alpha_{j-1}, \alpha_{j}}^{i_{j}}
$$

and $\omega \in \Omega_{T}^{i-2 j}$. Thus $d \varphi=(-1)^{j} F \wedge d \omega$. This finishs the proof of the proposition.

\section{Chern-Simons Classes and the Griffiths group}

5.1. Our objective in this section is to investigate the vanishing of the class $w_{n}(E, \nabla)$ for a flat bundle $E$ on a smooth, projective variety $X$ over $\mathbb{C}$. We will show that $w_{n}=0$ if and only if the $n$-th chern class $c_{n}(E)$ vanishes in a "generalized Griffiths group" $\operatorname{Griff}^{n}(X)$.

Let $X$ be a smooth, quasi-projective variety over $\mathbb{C}$. For $Z \subset X$ a closed subvariety and $A$ an abelian group, we write $H_{Z}^{*}(X, A)$ for the singular cohomology with supports in $Z$ and values in $A$. We write

$$
H_{\mathcal{Z}^{n}}^{*}(X, A)=\lim _{\longrightarrow} Z \subset X \text { cod. }{ }_{n} H_{Z}^{*}(X, A) .
$$


Purity implies that for $Z$ irreducible of codimension $n$,

$$
H_{Z}^{p}(X, A)= \begin{cases}0 & p<2 n \\ A(-n) & p=2 n\end{cases}
$$

Here $\mathbb{Z}(n)=(2 \pi i)^{n} \mathbb{Z}$ and $A(n):=A \otimes \mathbb{Z}(n)$. As a consequence

$$
H_{\mathcal{Z}^{n}}^{p}(X, \mathbb{Z}(n))= \begin{cases}0 & p<2 n \\ \mathcal{Z}^{n}(X) & p=2 n\end{cases}
$$

where $\mathcal{Z}^{n}(X)$ is the group of codimension $n$ algebraic cycles on $X$.

For $m<n$, define the Chow group of codimension $n$ algebraic cycles modulo codimension $m$ equivalence by

$$
C H_{m}^{n}(X):=\operatorname{Image}\left(\mathcal{Z}^{n}(X)=H_{\mathcal{Z}^{n}}^{2 n}(X, \mathbb{Z}(n)) \rightarrow H_{\mathcal{Z}^{m}}^{2 n}(X, \mathbb{Z}(n)) .\right.
$$

Of course, $C H_{0}^{*}(X)$ is the group of cycles modulo homological equivalence. It follows from [2] (7.3) that $\mathrm{CH}_{n-1}^{n}(X)$ is the group of codimension $n$ algebraic cycles modulo algebraic equivalence.

Definition 5.1.1. The generalized Griffiths group $\operatorname{Griff}^{n}(X)$ is defined to be the kernel of the map $C H_{1}^{n}(X) \rightarrow C H_{0}^{n}(X)$. In words, the generalized Griffiths group consists of cycles homologous to 0 on $X$ modulo those homologous to 0 on some divisor in $X$.

Example 5.1.2. Griff $^{2}(X)$ is the usual Griffiths group of codimension 2 cycles homologous to zero modulo algebraic equivalence.

5.2. With notation as above, let $\mathcal{H}^{p}(A)$ denote the Zariski sheaf on $X$ associated to the presheaf $U \mapsto H^{p}\left(U_{\text {an }}, A\right)$, cohomology for the classical (analytic) topology with coefficients in $A$. The principal object of study in [2] was a spectral sequence

$$
E_{2}^{p, q}(A)=H^{p}\left(X_{\mathrm{Zar}}, \mathcal{H}^{q}(A)\right) \Rightarrow H^{p+q}\left(X_{\mathrm{an}}, A\right) .
$$

associated to the "continuous" map $X_{\text {an }} \rightarrow X_{\text {Zar }}$. This spectral sequence was shown to coincide from $E_{2}$ onward with the "coniveau" spectral sequence

$$
E_{1}^{p, q}(A)=\bigoplus_{x \in \mathcal{Z}^{p}-\mathcal{Z}^{p+1}} H^{q-p}(x, A) \Rightarrow H^{p+q}\left(X_{\mathrm{an}}, A\right) .
$$

As a consequence of a Gersten resolution for the sheaves $\mathcal{H}^{p}(A)$, one had

$$
\begin{gathered}
H^{p}\left(X_{\mathrm{Zar}}, \mathcal{H}^{q}(A)\right)=(0) \text { for } p>q \\
H^{n}\left(X_{\mathrm{Zar}}, \mathcal{H}^{n}(\mathbb{Z}(n))\right) \cong C H_{n-1}^{n}(X) .
\end{gathered}
$$


The $E_{\infty}$-filtration $N^{*} H^{*}\left(X_{\mathrm{an}}, A\right)$ is the filtration by codimension,

$$
N^{p} H^{*}\left(X_{\text {an }}, A\right)=\text { Image }\left(H_{\mathcal{Z}^{p}}^{*}\left(X_{\mathrm{an}}, A\right) \rightarrow H^{*}\left(X_{\mathrm{an}}, A\right)\right) .
$$

Proposition 5.3.1. With notation as above, there is an exact sequence

$$
0 \rightarrow H^{2 n-1}\left(X_{\mathrm{an}}, \mathbb{Z}(n)\right) / N^{1} \rightarrow E_{n}^{0,2 n-1} \stackrel{d_{n}}{\longrightarrow} \operatorname{Griff}^{n}(X) \rightarrow 0 .
$$

Proof. It follows from (5.2.3) that we have

$$
\begin{gathered}
H^{2 n-1}\left(X_{\mathrm{an}}, \mathbb{Z}(n)\right) \rightarrow E_{\infty}^{0,2 n-1}=E_{n+1}^{0,2 n-1} \subset E_{n}^{0,2 n-1} \subset \cdots \\
\subset E_{2}^{0,2 n-1}=\Gamma\left(X, \mathcal{H}^{2 n-1}(\mathbb{Z}(n))\right)
\end{gathered}
$$

and

$$
C H_{n-1}^{n}(X)=E_{2}^{n, n} \rightarrow E_{3}^{n, n} \rightarrow \cdots \rightarrow E_{n+1}^{n, n}=E_{\infty}^{n, n} \subset H^{2 n}\left(X_{\mathrm{an}}, \mathbb{Z}(n)\right) .
$$

In fact, $E_{r}^{n, n} \cong C H_{n+1-r}^{n}(X)$. In particular, $E_{n}^{n, n} \cong C H_{1}^{n}(X)$. To see this, one can, for example, use the theory of exact couples [13 pp. 232 ff. One gets an exact triangle

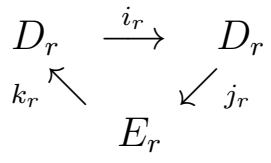

where in the appropriate degree

$$
D_{r}=\operatorname{Image}\left(H_{\mathcal{Z}^{n}}^{2 n}(X, \mathbb{Z}(n)) \rightarrow H_{\mathcal{Z}^{n-r+1}}^{2 n}(X, \mathbb{Z}(n))\right) \cong C H_{n-r+1}^{n}(X),
$$

$i_{r}=0$ and $j_{r}$ is an isomorphism.

The spectral sequence (5.2.1) now yields a diagram with exact rows, proving the proposition.

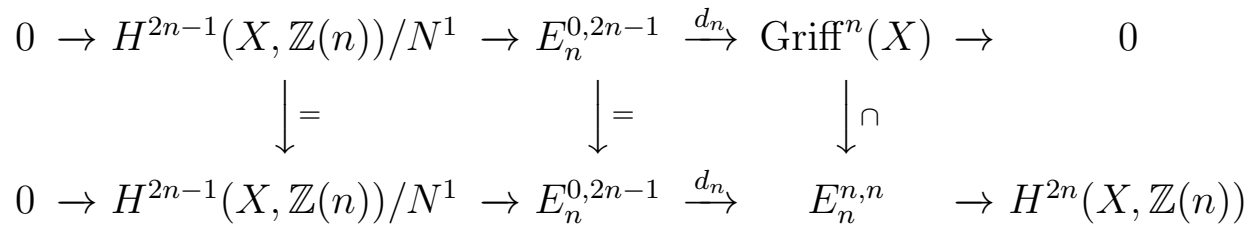

Proposition 5.4.1. Let $X$ be smooth and quasi-projective over $\mathbb{C}$. Let $(E, \nabla)$ be a vector bundle with an integrable connection on $X$. Let $n \geq 2$ be given, and let $d_{n}$ be as in (5.3.4). Let $c_{n}(E)$ be the $n$-th Chern class in $\operatorname{Griff}^{n}(X) \otimes \mathbb{Q}$. Then

(i) $w_{n}(E, \nabla) \in E_{n}^{0,2 n-1}(\mathbb{C}) \subset \Gamma\left(X, \mathcal{H}^{2 n-1}(\mathbb{C})\right)$.

(ii) $d_{n}\left(w_{n}\right)=c_{n}(E)$. 
Proof. The spectral sequence (5.2.1) in the case $A=\mathbb{C}$ coincides with the "second spectral sequence" of hypercohomology for

$$
H^{*}\left(X_{\mathrm{an}}, \mathbb{C}\right) \cong \mathbb{H}^{*}\left(X_{\mathrm{Zar}}, \Omega_{X / \mathbb{C}}^{*}\right)
$$

This is convenient for calculating the differentials in (5.2.1). Namely, we consider the complexes for $m \geq n$

$$
\begin{aligned}
\tau_{n, n} \Omega^{*} & :=\mathcal{H}^{n}(\mathbb{C})[-n] \\
\tau_{m, n} \Omega^{*} & :=\left(\Omega_{X}^{m} / d \Omega_{X}^{m-1} \rightarrow \ldots \rightarrow \Omega_{X}^{n-1} \rightarrow \Omega_{\text {closed }}^{n}\right)[-m] ; m<n
\end{aligned}
$$

We have maps

$$
\tau_{0, n} \Omega^{*} \rightarrow \tau_{1, n} \Omega^{*} \rightarrow \cdots \rightarrow \tau_{n, n} \Omega^{*} \rightarrow \tau_{n, n+1} \Omega^{*} \rightarrow \cdots \rightarrow \tau_{n, \infty} \Omega^{*},
$$

and

$$
\begin{gathered}
E_{r}^{0,2 n-1}=\operatorname{Image}\left(H^{2 n-1}\left(X, \tau_{2 n-r+1,2 n-1} \Omega^{*}\right) \rightarrow\right. \\
\left.H^{2 n-1}\left(X, \tau_{2 n-1,2 n-1} \Omega^{*}\right)=\Gamma\left(X, \mathcal{H}^{2 n-1}\right)\right) .
\end{gathered}
$$

There is a diagram of complexes

$$
\begin{aligned}
& {\left[\mathcal{K}_{n}^{m} \rightarrow \Omega^{n} \rightarrow \cdots \rightarrow \Omega^{2 n-2} \rightarrow \Omega_{\text {closed }}^{2 n-1}\right] \stackrel{a}{\rightarrow} \quad \Omega^{\infty} \mathcal{K}_{n}^{m}} \\
& \tau_{n+1,2 n-1} \Omega^{*}[n-1] \quad \stackrel{e}{\rightarrow} \tau_{2 n-1, \infty} \Omega^{*}[n-1],
\end{aligned}
$$

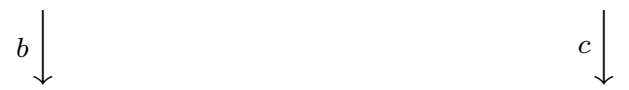

where $\Omega^{\infty} \mathcal{K}_{n}^{m}$ is the complex $\mathcal{K}_{n}^{m} \rightarrow \Omega^{n} \rightarrow \Omega^{n+1} \rightarrow \cdots$. We have

$$
c_{n}(E, \nabla) \in \mathbb{H}^{n}\left(X, \Omega^{\infty} \mathcal{K}_{n}^{m}\right)
$$

$$
c\left(c_{n}(E, \nabla)\right)=w_{n}(E, \nabla) \in \mathbb{H}^{2 n-1}\left(X, \tau_{2 n-1, \infty} \Omega^{*}\right) \cong H^{0}\left(X, \mathcal{H}^{2 n-1}\right) .
$$

The map $a$ is the inclusion of a subcomplex, and the quotient has no cohomology sheaves in degrees $<n+1$, so $a$ is an isomorphism on hypercohomology in degree $n$. It follows that $w_{n}(E, \nabla)$ lies in the image of the map $e$ in (5.4.3). By (5.4.2), this image is $E_{n}^{0,2 n-1}$.

To verify $d_{n}\left(w_{n}\right)=c_{n}(E)$, write $\Omega^{\infty} \mathcal{K}_{n}^{m}$ for the complex

$$
\mathcal{K}_{n}^{m} \rightarrow \Omega^{n} / d \Omega^{n-1} \rightarrow \Omega^{n+1} \rightarrow \cdots,
$$

and let $\bar{c}_{n}(E, \nabla) \in \mathbb{H}^{n}\left(X, \bar{\Omega}^{\infty} \mathcal{K}_{n}^{m}\right)$ be the image of $c_{n}(E, \nabla)$. Consider the distinguished triangle of complexes

$$
\tau_{n, 2 n-2} \Omega^{*}[n-1] \rightarrow \bar{\Omega}^{\infty} \mathcal{K}_{n}^{m} \stackrel{\alpha}{\longrightarrow} \mathcal{K}_{n}^{m} \oplus \tau_{2 n-1, \infty} \Omega^{*}[n-1]
$$

We have by definition $\alpha\left(\bar{c}_{n}(E, \nabla)\right)=\left(c_{n}(E), w_{n}(E)\right)$, so, writing $\partial$ for the boundary map,

$$
\partial\left(c_{n}(E)\right)=-\partial\left(w_{n}(E, \nabla)\right) \in \mathbb{H}^{2 n}\left(X, \tau_{n, 2 n-2} \Omega^{*}\right) .
$$


Note that the boundary map on $\mathcal{K}_{n}^{m}$ factors through the dlog map $\mathcal{K}_{n}^{m} \rightarrow \mathcal{H}^{n}$. Thus $\partial c_{n}(E)$ is the image of the Chern class. On the other hand, by (5.2.3) we have

$$
\mathbb{H}^{2 n}\left(X, \Omega^{*}\right) \rightarrow \mathbb{H}^{2 n}\left(X, \tau_{n, \infty} \Omega^{*}\right),
$$

from which it follows by standard spectral sequence theory that the image of the map

$$
H^{2 n}\left(X, \tau_{n, n} \Omega^{*}\right) \rightarrow \mathbb{H}^{2 n}\left(X, \tau_{n, 2 n-2} \Omega^{*}\right)
$$

coincides with $E_{n}^{n, n}$, and that the boundary map

$$
\delta: \Gamma\left(X, \mathcal{H}^{2 n-1}\right) \cong \mathbb{H}^{2 n-1}\left(X, \tau_{2 n-1, \infty} \Omega^{*}\right) \rightarrow \mathbb{H}^{2 n}\left(X, \tau_{n, 2 n-2} \Omega^{*}\right)
$$

coincides with $d_{n}$ from the statement of the proposition on $\delta^{-1}\left(E_{n}^{n, n}\right)=$ $E_{n}^{0,2 n-1}$. This completes the proof of the proposition.

Our next objective is to realize the sequence (5.3.1) as an exact sequence of mixed Hodge structures. To avoid complications, we replace $\mathbb{Z}$ with $\mathbb{Q}$ throughout. More precisely, we work with filtering direct limits of finite dimensional $\mathbb{Q}$-mixed Hodge structures, where the transition maps are maps of mixed Hodge structures.

Lemma 5.4.2. The spectral sequence (5.2.1) with $A=\mathbb{Q}(n)$ can be interpreted as a spectral sequence in the category of mixed Hodge structures.

Proof. The spectral sequence (5.2.2) can be deduced from an exact couple ([2],p.188)

$$
\cdots \rightarrow H_{\mathcal{Z}^{p}}^{p+q}(X, \mathbb{Q}(n)) \rightarrow H_{\mathcal{Z}^{p-1}}^{p+q}(X, \mathbb{Q}(n)) \rightarrow H_{\mathcal{Z}^{p-1} / \mathcal{Z}^{p}}^{p+q}(X, \mathbb{Q}(n)) \rightarrow \cdots
$$

These groups clearly have infinite dimensional mixed Hodge structures and the maps are morphisms of mixed Hodge structures. The lemma follows easily, since (5.2.1) coincides with the above from $E_{2}$ onward.

Remark 5.4.3. The groups $E_{r}^{n, n}$ are all quotients of

$$
H_{\mathcal{Z}^{n} / \mathcal{Z}^{n+1}}^{2 n}(X, \mathbb{Q}(n)) \cong \bigoplus_{z \in X^{n}} \mathbb{Q}
$$

so these groups all have trivial Hodge structures.

Proposition 5.5.1. The Chern-Simons class $w_{n}(E, \nabla) \in E_{n}^{0,2 n-1}(\mathbb{C})$ lies in $F^{0}$ (zeroeth piece of the Hodge filtration) for the Hodge structure defined by $E_{n}^{0,2 n-1}(\mathbb{Q}(n))$. 
Proof. We have $E_{n}^{0,2 n-1} \subset E_{2}^{0,2 n-1} \subset H^{2 n-1}(\mathbb{C}(X), \mathbb{C})$, where the group on the right is defined as the limit over Zariski open sets. Thus, it suffices to work "at the generic point". Let $\mathcal{S}$ denote the category of triples $(U, Y, \pi)$ with $Y$ smooth and projective, $\pi: Y \rightarrow X$ a birational morphism of schemes, and $U \subset Y$ Zariski open such that $Y_{U}$ is a divisor with normal crossings and $U \rightarrow \pi(U)$ is an isomorphism. Using resolution of singularities, one sees easily that

$$
H^{n}(\operatorname{Spec}(\mathbb{C}(X)), \mathbb{C}) \cong \lim _{\mathcal{S}} \mathbb{H}^{n}\left(Y, \Omega_{Y}^{*}(\log (Y-U))\right) .
$$

The Hodge filtration on the left is induced in the usual way from the first spectral sequence of hypercohomology on the right.

Lemma 5.5.2. For $\alpha \in \mathcal{S}$ let $j_{\alpha}: U_{\alpha} \hookrightarrow Y_{\alpha}$ be the inclusion. Then

$$
\lim _{\longrightarrow} \mathcal{S} H^{n}\left(Y_{\alpha}, j_{\alpha *} \mathcal{K}_{n, U_{\alpha}}^{m}\right)=(0), n \geq 1
$$

Proof of lemma. Given $j: U \hookrightarrow Y$ in $\mathcal{S}$ and $z \in H^{n}\left(Y, j_{*} \mathcal{K}_{n, U}^{m}\right)$, let $k: \operatorname{Spec}(\mathbb{C}(X)) \rightarrow Y$ be the generic point. We have $H^{n}\left(Y, k_{*} \mathcal{K}_{n, \mathbb{C}(X)}^{m}\right)=$ (0) since the sheaf is constant, so there exists $V \subset U$ open of finite type such that writing $\ell: V \rightarrow Y$, z dies in $H^{n}\left(Y, \ell_{*} \mathcal{K}_{n, V}^{m}\right)$. Let $m: V \rightarrow Z$ represent an object of $\mathcal{S}$ with $Z$ dominating $Y$. We have a triangle

$$
\begin{array}{cccc}
H^{n}\left(Y, j_{*} \mathcal{K}_{n, U}^{m}\right) & \rightarrow & H^{n}\left(Z, m_{*} \mathcal{K}_{n, V}^{m}\right) \\
\searrow & & \nearrow \\
& H^{n}\left(Y, \ell_{*} \mathcal{K}_{n, V}^{m}\right) &
\end{array}
$$

from which it follows that $z \mapsto 0$ in $\lim _{\mathcal{S}} H^{n}\left(Y_{\alpha}, j_{\alpha, *} \mathcal{K}_{n, U_{\alpha}}^{m}\right)$.

Returning to the proof of proposition 5.5.1, write $D_{\alpha}=Y_{\alpha}-U_{\alpha}$ for $\alpha \in \mathcal{S}$. We see from the lemma that the map labeled $a$ below is surjective:

$$
\begin{gathered}
c_{n}(E, \nabla) \in \mathbb{H}^{n}\left(X, \Omega^{\infty} \mathcal{K}_{n}^{m}\right) \\
\downarrow \\
\lim _{\longrightarrow} \mathcal{S} \mathbb{H}^{n}\left(Y_{\alpha}, j_{\alpha, *} \mathcal{K}_{n, U_{\alpha}}^{m} \rightarrow \Omega^{n}\left(\log \left(D_{\alpha}\right)\right) \rightarrow \cdots\right) \\
\nearrow a \\
\stackrel{\downarrow}{\longrightarrow} \mathcal{L} \mathbb{H}^{n-1}\left(Y_{\alpha}, \Omega^{n}\left(\log \left(D_{\alpha}\right)\right) \rightarrow \cdots\right) \stackrel{b}{\longrightarrow} H^{2 n-1}(\operatorname{Spec}(\mathbb{C}(X)), \mathbb{C})
\end{gathered}
$$

Since the image of $b$ is $F^{0}$ for the Hodge filtration on $H^{2 n-1}(\operatorname{Spec}(\mathbb{C}(X)), \mathbb{C})$, and since the composition of vertical arrows maps $c_{n}(E, \nabla)$ to the restriction of $w_{n}(E, \nabla)$ at the generic point, the proposition is proved.

Proposition 5.6.1. The Chern-Simons class $w_{n}(E, \nabla) \in E_{n}^{0,2 n-1}(\mathbb{Q}(n))$. 
Proof. The following diagram is commutative

$$
\begin{aligned}
& \mathbb{H}^{n}\left(X, \Omega^{\infty} \mathcal{K}_{n}^{m}\right) \rightarrow H^{0}\left(X, \mathcal{H}^{2 n-1}(\mathbb{C})\right) \rightarrow H^{2 n-1}(\operatorname{Spec}(\mathbb{C}(X)), \mathbb{C}) \\
& \downarrow \varphi(3.9 .1(3)) \quad \downarrow \\
& H^{2 n-1}\left(X_{\text {an }}, \mathbb{C} / \mathbb{Z}(n)\right) \longrightarrow H^{2 n-1}(\operatorname{Spec}(\mathbb{C}(X)), \mathbb{C} / \mathbb{Z}(n))
\end{aligned}
$$

We know from [7] and proposition 3.10.1 above that

$$
\varphi\left(c_{n}(E, \nabla)\right)=c_{n}^{\text {an }}(E, \nabla),
$$

and, using the deep theorem of Reznikov [18], that this class is torsion. In particular, the image of $c_{n}(E, \nabla)$ on the upper right lies in $H^{2 n-1}(\operatorname{Spec}(\mathbb{C}(X)), \mathbb{Q}(n))$. As a matter of fact, in 5.6.2, we will only use that $w_{n}(E, \nabla) \in E_{n}^{0,2 n-1}(\mathbb{R}(n))$. For this we don't need the full strength of [18], but only that $c_{n}^{\text {an }}(E, \nabla)=0 \in H^{2 n-1}\left(X_{\text {an }}, \mathbb{C} / \mathbb{R}(n)\right)$, which is a consequence of Simpson's theorem [19] asserting that $(E, \nabla)$ deforms to a $\mathbb{C}$ variation of Hodge structure.

Theorem 5.6.2. Let $X$ be a smooth, projective variety over $\mathbb{C}$. Let $E$ be a vector bundle on $X$, and let $\nabla$ be an integrable connection on $E$. Then $w_{n}(E, \nabla) \in H^{0}\left(X, \mathcal{H}^{2 n-1}(\mathbb{C})\right)$ vanishes if and only if the cycle class $c_{n}(E)$ is trivial in $\operatorname{Griff}^{n}(X) \otimes \mathbb{Q}$.

Proof. Consider the exact sequence of mixed Hodge structures

$$
0 \rightarrow H^{2 n-1}\left(X_{\mathrm{an}}, \mathbb{Q}(n)\right) / N^{1} \rightarrow E_{n}^{0,2 n-1}(\mathbb{Q}(n)) \rightarrow \operatorname{Griff}^{n}(X) \otimes \mathbb{Q} \rightarrow 0 .
$$

Write $H$ for the group on the left. It is pure of weight -1 , so $H(\mathbb{Q}) \cap F^{0} H(\mathbb{C})=(0)$. It follows that $w_{n}(E, \nabla)=0$ if and only if its image $c_{n}(E)$ in $\operatorname{Griff}^{n}(X) \otimes \mathbb{Q}$ vanishes.

The following corollary is a simple application of the theorem to the example (0.2) discussed in the introduction.

Corollary 5.6.3. Let $E, X, \nabla$ be as above. Assume $E$ has rank 2, and that the determinant bundle is trivial, with the trivial connection. Let $U \subset X$ be affine open such that $E \mid U$ is trivial, and let $\left(\begin{array}{cc}\alpha & \beta \\ \gamma & -\alpha\end{array}\right)$ be the connection matrix. Then $c_{2}(E) \otimes \mathbb{Q}$ is algebraically equivalent to 0 on $X$ if and only if there exists a meromorphic 2-form $\eta$ on $X$ satisfying $d \eta=\alpha \wedge d \alpha=\alpha \wedge \beta \wedge \gamma$.

\section{Logarithmic Poles}

In this section we consider a normal crossing divisor $D \subset X$ on a smooth variety $X$, the inclusion $j: X-D \rightarrow X$, and a bundle $E$, together with a flat connection $\nabla: E \rightarrow \Omega_{X}^{1}(\log D) \otimes E$ with $\log$ arithmic 
poles along $D$. The characteristic of the ground field $k$ is still 0 . Finally recall from [2] that one has an exact sequence

$$
0 \rightarrow H^{0}\left(X, \mathcal{H}^{j}\right) \rightarrow H^{0}\left(X-D, \mathcal{H}^{j}\right) \rightarrow \oplus_{i} \operatorname{res} H^{0}\left(k\left(D_{i}\right), \mathcal{H}^{j-1}\right)
$$

Theorem 6.1.1. Let $(E, \nabla, D)$ be a flat connection with logarithmic poles. Then

$$
w_{n}(E, \nabla) \in H^{0}\left(X, \mathcal{H}^{2 n-1}\right) \subset H^{0}\left(X-D, \mathcal{H}^{2 n-1}\right)=H^{0}\left(X, j_{*} \mathcal{H}^{2 n-1}\right) .
$$

Proof. By 6.0.3, one just has to compute the residues of $w_{n}(E, \nabla)$ along generic points of $D$. So one may assume that the local equation of $\nabla$ is $A=B \frac{d x}{x}+C$, where $B$ is a matrix of regular functions, $x$ is the local equation of a smooth component of $D$, and $C$ is a matrix of regular one forms. Furthermore, as $d A=A^{2}=\frac{1}{2}[A, A]$, the formulae of theorem 2.2.1 say that the local shape of $w_{n}(E, \nabla)$ is $\operatorname{Tr} \lambda A(d A)^{n-1}=$ $\lambda \operatorname{Tr}\left(B \frac{d x}{x}+C\right)\left(d B \frac{d x}{x}+d C\right)^{n-1}$ for some $\lambda \in \mathbb{Q}$. So upto coefficient one has to compute

$$
\begin{gathered}
\operatorname{Tr} \operatorname{Res}\left(B \frac{d x}{x}+C\right)\left((d C)^{n-1}+\sum_{a+b=n-2}(d C)^{a} d B \frac{d x}{x}(d C)^{b}\right)= \\
\operatorname{Tr} \operatorname{Res}\left[C \sum_{a+b=n-2}(d C)^{a} d B(d C)^{b}+B(d C)^{n-1}\right] \frac{d x}{x} .
\end{gathered}
$$

On the other hand, the integrability condition reads

$$
(d B-(C B-B C)) \frac{d x}{x}+d C-C^{2}=0,
$$

from which one deduces

$$
\begin{gathered}
d C \frac{d x}{x}=C^{2} \frac{d x}{x} \\
\operatorname{Res}(d B-(C B-B C)) \frac{d x}{x}=0
\end{gathered}
$$

Applying (6.1.3) to (6.1.2), we reduce to calculating

$$
\operatorname{Tr} \operatorname{Res}\left[\sum_{a+b=n-2}(d C)^{a} C d B(d C)^{b}+B(d C)^{n-1}\right] \frac{d x}{x}
$$

Since we are only interested in calculating (6.1.5) modulo exact forms, we can use $d(C B)=d C B-C d B$ and move copies of $d C$ to the right in $(6.1 .5)$ under the trace. The problem becomes to show

$$
\operatorname{Tr} \operatorname{Res} B(d C)^{n-1} \frac{d x}{x}
$$


is exact. It follows from (6.1.4) that

$$
\operatorname{Tr} \operatorname{Res} C^{2 n-3} d B \frac{d x}{x}=\operatorname{Tr} \operatorname{Res}\left[C^{2 n-2} B-C^{2 n-3} B C\right] \frac{d x}{x}
$$

Bringing the $C$ to the left in the last term changes the sign, so we get by $(6.1 .3)$

$\operatorname{Tr} \operatorname{Res}(d C)^{n-2} C d B \frac{d x}{x}=\operatorname{Tr} \operatorname{Res} C^{2 n-3} d B \frac{d x}{x}=\operatorname{Tr} \operatorname{Res} 2(d C)^{n-1} B \frac{d x}{x}$.

Thus

$$
\begin{array}{r}
\operatorname{Tr} \operatorname{Res}(d C)^{n-1} B \frac{d x}{x}=\operatorname{Tr} \operatorname{Res}(d C)^{n-2}(C d B-d C B) \frac{d x}{x}= \\
-\operatorname{Tr} \operatorname{Res} d\left[C(d C)^{n-3} d(C B) \frac{d x}{x}\right] .
\end{array}
$$

This form is exact, so we are done.

6.2. We want now to understand the image of $w_{n}(E, \nabla)$ under the map $d_{n}$ defined in 5.3.1. Of course 5.4.1 says that $d_{n}\left(w_{n}((E, \nabla) \mid(X-\right.$ $D)))=c_{n}(E)$.

Definition 6.2.1. (see [10], Appendix B): Let $(E, \nabla)$ be a flat connection with logarithmic poles along $D$, with residue

$$
\Gamma=\oplus \Gamma_{s} \in \oplus_{s} H^{0}\left(D_{s}, \text { End }\left.E\right|_{D_{s}}\right) .
$$

One defines

$$
\begin{gathered}
N_{i}^{C H}(\Gamma)=(-1)^{i} \sum_{\alpha_{1}+\cdots+\alpha_{s}=i}\left(\begin{array}{c}
i \\
\alpha
\end{array}\right) \operatorname{Tr}\left(\Gamma_{1}^{\alpha_{1}} \circ \cdot \ldots \cdot \circ \Gamma_{s}^{\alpha_{s}}\right) \cdot\left[D_{1}\right]^{\alpha_{1}} \cdots\left[D_{s}\right]^{\alpha_{s}} \\
\quad \in C H^{i}(X) \otimes \mathbb{C} .
\end{gathered}
$$

One defines as usual the corresponding symmetric functions $c_{i}^{C H}(\Gamma) \in$ $C H^{i}(X) \otimes \mathbb{C}$ as polynomial with $\mathbb{Q}$ coefficients in the Newton functions $N_{i}^{C H}(\Gamma)$. For example

$$
\begin{gathered}
c_{2}^{C H}(\Gamma)=\frac{1}{2}\left[\left(\sum_{s} \operatorname{Tr}\left(\Gamma_{s}\right) \cdot D_{s}\right)^{2}-\right. \\
\left.2\left(\sum_{s} \operatorname{Tr}\left(\Gamma_{s} \cdot \Gamma_{s}\right) \cdot D_{s}^{2}+2 \sum_{s<t} \operatorname{Tr}\left(\Gamma_{s} \cdot \Gamma_{t}\right) D_{s} \cdot D_{t}\right)\right] \\
\in C H^{2}(X) \otimes \mathbb{C} .
\end{gathered}
$$

We denote by $c_{2}(\Gamma)$ its image in $H^{2}\left(X, \Omega_{X, c l}^{2}\right)$ and also by $c_{2}(\Gamma)$ its image in $H^{2}\left(X, \mathcal{H}_{D R}^{2}\right)$. 
Note that these invariants vanish when the connection has nilpotent residues $\Gamma_{s}$. (This condition forces the local monodromies around the components of $D$ to be unipotent (see [5])).

Theorem 6.2.2. Assume $k$ has characteristic zero and $X$ is proper. Then

$$
c_{2}(E)-c_{2}(\Gamma)=d_{2}\left(w_{2}(E, \nabla)\right) \in H^{2}\left(X, \mathcal{H}^{2}\right) .
$$

Proof. In order to simplify the notations, we denote by $c_{2}(\Gamma)$ the same expression in $C H^{2}(X) \otimes \mathbb{C}, \oplus_{s} C H^{1}\left(D_{s}\right) \otimes \mathbb{C}, \oplus_{s} F^{1} H_{D R}^{2}\left(D_{s}\right)$ etc., where we always distribute $2 D_{s} \cdot D_{t}$ for $s<t$ as one $D_{s} \cdot D_{t}$ on $D_{s}$ and one on $D_{t}$.

We denote by $\pi: Q \rightarrow X$ the flag bundle of $E$. As $\pi^{*}$ induces an isomorphism

$$
\frac{H^{2}\left(X, d \Omega_{X}^{1}\right)}{H^{1}\left(X, \mathcal{H}^{2}\right)}=\frac{H^{3}\left(X, \mathcal{O}_{X} \rightarrow \Omega_{X}^{1}\right)}{N^{1} H^{3}(X)} \stackrel{\sim}{\longrightarrow} \frac{H^{3}\left(Q, \mathcal{O}_{Q} \rightarrow \Omega_{Q}^{1}\right)}{N^{1} H^{3}(Q)}
$$

and an injection $H^{2}\left(X, \mathcal{H}^{2}\right) \rightarrow H^{2}\left(Q, \mathcal{H}^{2}\right)$, it is enough to prove the compatibility on $Q$ via the exact sequence ([2])

$$
0 \rightarrow \frac{H^{3}\left(X, \mathcal{O}_{X} \rightarrow \Omega_{X}^{1}\right)}{N^{1} H^{3}(X)} \rightarrow H^{2}\left(X, \Omega_{X, \mathrm{clsd}}^{2}\right) \rightarrow H^{2}\left(X, \mathcal{H}^{2}\right)
$$

Write $D^{\prime}{ }_{s}=\pi^{*} D_{s}$, and consider $\left(\mathcal{O}\left(D_{s}^{\prime}\right), \nabla_{s}\right) \in \mathbb{H}^{1}\left(Q, \mathcal{K}_{1} \rightarrow \Omega_{Q}^{1}\left(\log D_{s}^{\prime}\right)_{\text {clsd }}\right)$, where $\nabla_{s}$ is the canonical connection with residue -1 along $D_{s}^{\prime}$.

We define a product

$$
\begin{aligned}
\left(\mathcal{K}_{i}^{m} \rightarrow\left(\pi^{*} \Omega_{X}^{i}(\log D)\right)_{\tau d}\right) \times\left(\mathcal{K}_{j}^{m} \rightarrow\left(\pi^{*} \Omega_{X}^{j}(\log D)\right)_{\tau d}\right) \\
\stackrel{\bullet}{\longrightarrow}\left(\mathcal{K}_{i+j}^{m} \rightarrow\left(\pi^{*} \Omega_{X}^{i+j}(\log D)\right)_{\tau d}\right)
\end{aligned}
$$

by

$$
x \cdot x^{\prime}= \begin{cases}x \cup x^{\prime} & \text { if } \operatorname{deg} x^{\prime}=0 \\ \tau d \log x \wedge x^{\prime} & \text { if } \operatorname{deg} x=0 \text { and } \operatorname{deg} x^{\prime}=1 \\ 0 & \text { otherwise }\end{cases}
$$

(Here $\tau d: \pi^{*} \Omega_{X}^{i}(\log D) \rightarrow \pi^{*} \Omega_{X}^{i+1}(\log D)$ comes from the splitting $\tau: \Omega_{Q}^{1}\left(\log D^{\prime}\right) \rightarrow \pi^{*} \Omega_{X}^{1}(\log D)$. See proposition 4.4.1] as well as [7] and [8].) One verifies that

$$
d\left(x \cdot x^{\prime}\right)=d x \cdot x^{\prime}+(-1)^{\operatorname{deg} x} x \cdot d x^{\prime},
$$

the only non trivial contribution left and right being for $\operatorname{deg} x=\operatorname{deg} x^{\prime}=$ 0 . 
This product defines elements ( $W_{1}$ is the weight filtration)

$\epsilon_{s t}=\left(\mathcal{O}\left(D_{s}^{\prime}\right), \nabla_{s}\right) \cdot\left(\mathcal{O}\left(D_{t}^{\prime}\right), \nabla_{t}\right) \in \mathbb{H}^{2}\left(Q, \mathcal{K}_{2} \rightarrow W_{1} \Omega_{Q}^{2}\left(\log \left(D_{s}^{\prime}+D_{t}^{\prime}\right)\right)_{c l}\right)$ which map to $D_{s}^{\prime} \cdot D_{t}^{\prime}$ in $C H^{2}(Q)$. Moreover Res $\epsilon_{s t}$ is the class of $D_{s}^{\prime} \cdot D_{t}^{\prime}$ sitting diagonally in

$$
F^{1} H_{D R}^{2}\left(D_{s}^{\prime}\right) \oplus F^{1} H_{D R}^{2}\left(D_{t}^{\prime}\right)
$$

if $s \neq t$; or in $F^{1} H_{D R}^{2}\left(D_{s}^{\prime}\right)$ if $s=t$.

Next we want to define a cocycle $N_{2}\left(\pi^{*}(E, \nabla)\right)$.

Let $h_{i j}(=h)$ be the upper triangular transition functions of $\left.E\right|_{Q}$ adapted to the tautological flag $E_{i}$, and write $B_{i}$ for the local connection matrix in $\Omega_{Q}^{1}\left(\log D^{\prime}\right), D^{\prime}=\pi^{-1} D$. Then $\tau B_{i}$ is upper triangular, and $\tau d B_{i}=d \tau B_{i}$ has zero's on the diagonal [7], (0.7), (2.7). Let

$$
w_{i}=\operatorname{Tr}\left(B_{i} d B_{i}\right) .
$$

Using $\operatorname{Tr}\left(d h h^{-1}\right)^{3}=0$, one computes that $w_{i}-w_{j}=-3 \operatorname{Tr} d\left(h^{-1} d h B_{j}\right)$. But

$$
\begin{aligned}
\operatorname{Tr} h^{-1} d h B_{j}=\operatorname{Tr} h^{-1} B_{i} h B_{j} \\
\operatorname{Tr} h_{i k}^{-1} d h_{i j} d h_{j k}=\operatorname{Tr} h_{i k}^{-1}\left(B_{i} h_{i j}-h_{i j} B_{j}\right)\left(B_{j} h_{j k}-h_{j k} B_{j}\right) \\
=\delta \operatorname{Tr}\left(B_{i} h B_{j} h^{-1}\right) .
\end{aligned}
$$

Here $\delta$ is the Cech coboundary. Writing $\mathcal{C}^{i}$ for Cech $i$-cochains, we may define

$$
\begin{aligned}
3 N_{2}\left(\pi^{*}(E, \nabla)\right) & =\left(3 \sum_{a=0}^{r} \xi_{i j}^{a} \cup \xi_{j k}^{a},-3 \operatorname{Tr}\left(h^{-1} d h B_{j}\right), w_{i}\right) \in \\
\left(\mathcal{C}^{2}\left(Q, \mathcal{K}_{2}\right)\right. & \left.\times \mathcal{C}^{1}\left(Q, \Omega_{Q}^{2}\left(\log D^{\prime}\right)\right) \times \mathcal{C}^{0}\left(Q, \Omega_{Q}^{3}\left(\log D^{\prime}\right)\right)\right)_{d+\delta}
\end{aligned}
$$

where $\left(\xi_{i j}^{1}, \ldots, \xi_{i j}^{r}\right)$ is the diagonal part of $h_{i j}$. This defines $3 N_{2}\left(\pi^{*}(E, \nabla)\right)$ as a class in $\mathbb{H}^{2}\left(Q, \mathcal{K}_{2} \rightarrow \Omega_{Q}^{2}\left(\log D^{\prime}\right) \rightarrow \ldots\right)$ which maps to

$$
\begin{gathered}
3 \tau N_{2}\left(\pi^{*}(E, \nabla)\right)=\left(3 \sum_{a=1}^{r} \xi_{i j}^{a} \cup \xi_{j k}^{a}, 3 \sum_{a=1}^{r} \omega_{i}^{a} \wedge\left(\delta \omega^{a}\right)_{i j}, 0\right) \\
\in \mathbb{H}^{2}\left(Q, \mathcal{K}_{2} \rightarrow \pi^{*} \Omega_{X}^{2}\left(\log D^{\prime}\right)_{\tau d}\right)
\end{gathered}
$$

where $\left(\omega_{i}^{1}, \ldots, \omega_{i}^{r}\right)$ is the diagonal part of $\tau B_{i}$.

As the image of $\tau N_{2}\left(\pi^{*}(E, \nabla)\right)$ in $H^{2}\left(Q, \mathcal{K}_{2}\right)$ is just the second Newton class of $E$, the argument of [8], (1.7) shows that

$$
N_{2}(E, \nabla):=\tau N_{2}\left(\pi^{*}(E, \nabla)\right) \in
$$

$$
\mathbb{H}^{2}\left(X, \mathcal{K}_{2} \rightarrow \Omega_{X}^{2}(\log D) \rightarrow \ldots\right) \subset \mathbb{H}^{2}\left(Q, \mathcal{K}_{2} \rightarrow \pi^{*} \Omega_{X}^{2}(\log D) \rightarrow \ldots\right)
$$


We observe that $w(B)=\operatorname{Tr} B d B \in W_{2} \Omega_{Q}^{3}\left(\log D^{\prime}\right)$ (weight filtration) so the cocycle

$$
\begin{gathered}
2 x=-N_{2}\left(\pi^{*}(E, \nabla)\right)+c_{1}\left(\pi^{*}(E, \nabla)\right)^{2}= \\
\left(-\operatorname{Tr}\left(h^{-1} d h\right)^{2}+\operatorname{Tr} h^{-1} d h \cdot \operatorname{Tr} h^{-1} d h,\right. \\
\left.\operatorname{Tr}\left(h^{-1} d h B\right)-\operatorname{Tr} h^{-1} d h \cdot \operatorname{Tr} B,-\frac{w(B)}{3}\right)
\end{gathered}
$$

defines a class in

$$
\mathbb{H}^{2}\left(Q, \Omega_{c l}^{2} \rightarrow W_{1} \Omega_{Q}^{2}\left(\log D^{\prime}\right) \rightarrow W_{2} \Omega_{Q}^{3}\left(\log D^{\prime}\right)_{c l}\right) .
$$

One has an exact sequence

$$
\begin{gathered}
0 \rightarrow \mathbb{H}^{2}\left(Q, \Omega_{c l}^{2} \rightarrow W_{1} \Omega_{Q}^{2}\left(\log D^{\prime}\right) \rightarrow W_{1} \Omega_{Q}^{3}\left(\log D^{\prime}\right)_{c l}\right) \\
\rightarrow \mathbb{H}^{2}\left(Q, \Omega_{c l}^{2} \rightarrow W_{1} \Omega_{Q}^{2}\left(\log D^{\prime}\right) \rightarrow W_{2} \Omega_{Q}^{3}\left(\log D^{\prime}\right)_{c l}\right) \\
\stackrel{\text { residue }}{\longrightarrow} \oplus_{s<t} H^{0}\left(D_{s t}^{\prime}, \Omega_{D_{s t, c l}^{\prime}}^{1}\right) .
\end{gathered}
$$

As $D_{s t}^{\prime}$ is proper smooth, one has

$$
H^{0}\left(D_{s t}^{\prime}, \Omega_{D_{s t, c l}^{\prime}}^{1}\right) \subset H^{0}\left(D_{s t}^{\prime}, \mathcal{H}^{1}\right)=H^{1}\left(D_{s t}^{\prime}\right) .
$$

The residue of $2 x$ along $D_{s t}^{\prime}$ is just the residue of $-\frac{1}{3} w(B)$ along $D_{s t}^{\prime}$ via

$$
\begin{gathered}
H^{0}\left(Q, \mathcal{H}^{3}\left(\log D^{\prime}\right)\right)=H^{0}\left(Q-D^{\prime}, \mathcal{H}^{3}\right) \\
\rightarrow \oplus_{s} H^{0}\left(D_{s}^{\prime}-\cup_{t \neq s} D_{t}^{\prime}, \mathcal{H}^{2}\right) \\
\rightarrow \oplus_{s<t} H^{0}\left(D_{s t}^{\prime}, \mathcal{H}^{1}\right),
\end{gathered}
$$

which vanishes. Therefore

$$
2 x \in \mathbb{H}^{2}\left(Q, \Omega_{c l}^{2} \rightarrow W_{1} \Omega_{Q}^{2}\left(\log D^{\prime}\right) \rightarrow W_{1} \Omega_{Q}^{3}\left(\log D^{\prime}\right)_{c l}\right) .
$$

Its residue in $\oplus_{s} H^{1}\left(D_{s}^{\prime}, \Omega_{D_{s}^{\prime}}^{1}\right)$ is $\left(\operatorname{Tr}\left(h^{-1} d h \cdot \Gamma\right)-\operatorname{Tr} h^{-1} d h \cdot \operatorname{Tr} \Gamma\right)$. By [10], Appendix B, one has $-h^{-1} d h=\sigma\left(D^{\prime}\right) \cdot \Gamma$ in $H^{1}\left(Q, \Omega_{Q}^{1} \otimes \operatorname{End} E\right)$ where $\sigma\left(D^{\prime}\right)$ is the extension

$$
0 \rightarrow \Omega_{Q}^{1} \rightarrow \Omega_{Q}^{1}\left(\log D^{\prime}\right) \rightarrow \oplus_{s} \mathcal{O}_{D_{s}^{\prime}} \rightarrow 0 .
$$

One has

1. $-D_{s}^{\prime} \cdot D_{s}^{\prime}$ is the push down extension of $\sigma\left(D_{s}^{\prime}\right)$ by $\Omega_{Q}^{1} \rightarrow \Omega_{D_{s}^{\prime}}^{1}$ in $H^{1}\left(Q, \Omega_{D_{s}^{\prime}}^{1}\right)$

2. $-D_{s}^{\prime} \cdot D_{t}^{\prime}$ is the extension

$$
0 \rightarrow \Omega_{D_{t}^{\prime}}^{1} \rightarrow \Omega_{D_{t}^{\prime}}^{1}\left(\log \left(D_{s}^{\prime} \cap D_{t}^{\prime}\right)\right) \rightarrow \mathcal{O}_{D_{s}^{\prime} \cap D_{t}^{\prime}} \rightarrow 0
$$

in $H^{1}\left(Q, \Omega_{D_{t}^{\prime}}^{1}\right)$. 
It follows that residue $x=c_{2}(\Gamma)$ in $\oplus_{s} H^{1}\left(D_{s}^{\prime}, \Omega_{D_{s}^{\prime}}^{1}\right)$.

For appropriate $\lambda_{s t} \in k$ (the coefficients of $\left.c_{2}(\Gamma)\right), c_{2}(\Gamma)=$ residue $\sum \lambda_{s t} \epsilon_{s t}$ in $\oplus_{s} H^{1}\left(D_{s}^{\prime}, \Omega_{D_{s}^{\prime}}^{1}\right)$. So one has

$$
\text { residue }\left(x-\sum \lambda_{s t} \epsilon_{s t}\right) \in \oplus_{s} F^{2} H^{2}\left(D_{s}^{\prime}\right)
$$

Again, since residue $\left(x-\sum \lambda_{s t} \epsilon_{s t}\right)=$ residue $x=0$ in $\oplus_{s} H^{0}\left(D_{s}^{\prime}, \mathcal{H}^{2}\right) \subset$ $\oplus_{s} H^{2}\left(k\left(D_{s}^{\prime}\right)\right)$, one has that in $\oplus_{s} F^{1} H^{2}\left(D_{s}^{\prime}\right)$

$$
\text { residue }\left(x-\sum \lambda_{s t} \epsilon_{s t}\right) \in \oplus_{s} F^{2} H^{2}\left(D_{s}^{\prime}\right) \cap H^{1}\left(D_{s}^{\prime}, \mathcal{H}^{1}\right)=0 \text {. }
$$

This shows that residue $\left(x-\sum \lambda_{s t} \epsilon_{s t}\right)=0$ in $\oplus_{s} F^{1} H^{2}\left(D_{s}^{\prime}\right)$, that is

$$
\begin{gathered}
w_{2}(E, \nabla)=\left(x-\sum \lambda_{s t} \epsilon_{s t}\right) \in \\
\frac{\mathbb{H}^{2}\left(Q, \Omega_{c l}^{2} \rightarrow \Omega^{2} \rightarrow \Omega_{c l}^{3}\right)=H^{0}\left(Q, \mathcal{H}^{3}\right)}{\operatorname{Im} \oplus_{s} H^{1}\left(D_{s}^{\prime}\right)}
\end{gathered}
$$

and maps to

$$
c_{2}(E)-c_{2}(\Gamma) \text { in } H^{2}\left(Q, \mathcal{H}^{2}\right)
$$

6.3. question. We know (see [10], Appendix B) that on $X$ proper, the image of $c_{n}(\Gamma)$ in the de Rham cohomology $H_{D R}^{2 n}(X)$ is the Chern class $c_{n}^{D R}(E)$. This inclines to ask whether

$$
c_{n}(E)-c_{n}(\Gamma)=d_{n}\left(w_{n}(E, \nabla)\right) \in \operatorname{Griff}^{n}(X) .
$$

\section{4 .}

Theorem 6.4.1. Let $(E, \nabla)$ be a flat connection with logarithmic poles along a normal crossing divisor $D$ on a smooth proper variety $X$ over $\mathbb{C}$. When $(E, \nabla) \mid(X-D)$ is a complex variation of Hodge structure, then $w_{2}(E, \nabla)=0$ if and only if $c_{2}(E)-c_{2}(\Gamma)=0 \in H^{2}\left(X, \mathcal{H}^{2}\right)$. When furthermore $(E, \nabla) \mid(X-D)$ is a Gauß-Manin system, then $w_{2}(E, \nabla) \in$ $H^{0}\left(X, \mathcal{H}^{3}(\mathbb{Q}(2))\right)$, and if it has nilpotent residues along the components of $D$, then $w_{2}(E, \nabla)=0$ if and only if $c_{2}(E)=0 \in H^{2}\left(X, \mathcal{H}^{2}\right)$.

Proof. As in proposition 5.5.1, one has $w_{n}(E, \nabla) \in F^{0}$. In fact, the proof does not use that $\nabla$ is everywhere regular, but only that $w_{n}(E, \nabla)$ comes from a class in $\mathbb{H}^{n}\left(Y, \mathcal{K}_{n}^{m} \rightarrow \Omega_{Y}^{n}(\log (Y-U) \rightarrow \ldots)\right.$ on some $(U, Y, \pi) \in \mathcal{S}$. Further, if $(E, \nabla) \mid(X-D)$ is a $\mathbb{C}$ variation of Hodge structure, then $w_{n}(E, \nabla) \in H^{0}\left(X, \mathcal{H}^{2 n-1}(\mathbb{R}(n))\right.$ as

$$
w_{n}(E, \nabla) \mid(X-D) \in H^{0}\left(X-D, \mathcal{H}^{2 n-1}(\mathbb{R}(n))\right)
$$


(see proof of proposition 5.6.1). When $(E, \nabla) \mid(X-D)$ is a Gauß-Manin system, then again one argues exactly as in the proof of proposition 5.6.1 using [4] to show $w_{2}(E, \nabla) \in H^{0}\left(X, \mathcal{H}^{3}(\mathbb{Q}(2))\right)$. Finally $c_{2}(\Gamma)=0$ when the residues of the connection are nilpotent.

\section{EXAmples}

7.1. Let $X$ be a good compactification of the moduli space of curves of genus $g$ with some level, such that a universal family $\varphi: \mathcal{C} \rightarrow$ $X$ exists. Let $(E, \nabla)$ be the Gauß-Manin system $R^{1} \varphi_{*} \Omega_{\mathcal{C} / X}^{\bullet}(\log \infty)$. Then Mumford [16], (5.3) shows that $c_{i}^{C H}(E) \otimes \mathbb{Q}=0$ in $C H^{i}(X) \otimes \mathbb{Q}$ for $i \geq 1$, so a fortiori $c_{i}(E) \otimes \mathbb{Q}=0$ in the Griffiths group. As $\nabla$ has nilpotent residues (the local monodromies at infinity of the GaußManin system are unipotent and $(E, \nabla)$ is Deligne's extension [5]), one applies theorem 6.4.1.

In particular, for any semi-stable family of curves $\varphi: Y \rightarrow X$ over a field $k$ of characteristic 0 ,

$$
w_{n}\left(R^{1} \varphi_{*} \Omega_{Y / X}^{\bullet}(\log \infty)\right)=0,
$$

for $n=2$ and for $n \geq 2$ if $\varphi$ is smooth (or if the question (6.3) has a positive response).

7.2. Let $X$ be a level cover of the moduli space of abelian varieties such that a universal family $\varphi: \mathcal{A} \rightarrow X$ exists. The Riemann-RochGrothendieck theorem applied to a principal polarization $L$ on $\mathcal{A}$ together with Mumford's theorem that

$$
\varphi_{*} L^{n}=M \otimes \text { trivial }
$$

for some rank 1 bundle $M$, imply that $c_{i}^{C H}(E) \otimes \mathbb{Q}=0$ for $E=$ $R^{1} \varphi_{*} \Omega_{\mathcal{A} / X}^{\bullet} \mid X_{0}$, where $X_{0}$ is the smooth locus of $\varphi$. This result was communicated to us by G. van der Geer [12]. In particular, for any smooth family $\varphi: Y \rightarrow X$ of abelian varieties with $X$ smooth proper over a field of characteristic $0, w_{n}\left(R^{1} \varphi_{*} \Omega_{Y / X}^{\bullet}\right)=0$ for all $n \geq 2$.

7.3. Let $\varphi: Y \rightarrow X$ be a smooth proper family of surfaces over $X$ smooth. The Riemann-Roch-Grothendieck theorem, as applied by Mumford in [16], implies that the Chern character verifies

$$
\operatorname{ch}\left(\sum_{i=0}^{i=4}(-1)^{i} R^{i} \varphi_{*} \Omega_{Y / X}^{\bullet}\right) \in C H^{0}(X) \otimes \mathbb{Q} \subset C H^{\bullet}(X) \otimes \mathbb{Q} .
$$

As $R^{1} \varphi_{*} \Omega_{Y / X}^{\bullet}$ is dual to $R^{3} \varphi_{*} \Omega_{Y / X}^{\bullet}$, the two previous examples show that $c_{i}\left(R^{2} \varphi_{*} \Omega_{Y / X}^{\bullet}\right)=0$ in $C H^{i}(X) \otimes \mathbb{Q}$ for $i \geq 1$. This implies $w_{n}\left(R^{2} \varphi_{*} \Omega_{Y / X}^{\bullet}\right)=0$ for all $n \geq 2$ when $X$ is proper. 
7.4. As shown in [9], $w_{n}(E, \nabla)=0$ in characteristic zero when $(E, \nabla)$ trivializes on a finite (not necessarly smooth) covering of $X$.

7.5. Let $\varphi: Y \rightarrow X$ be a smooth proper family defined over a perfect field $k$ of sufficiently large characteristic. Let $(E, \nabla)$ be the GaußManin system $R^{a} \varphi_{*} \Omega_{Y / X}^{\bullet}$. Consider $w_{n}(E, \nabla) \in H^{0}\left(X, \mathcal{H}^{2 n-1}\right)$, which is the restriction of the corresponding class in characteristic zero when $\varphi$ comes from a smooth proper family in characteristic zero. Assume this. As $H^{0}\left(X, \mathcal{H}^{2 n-1}\right) \subset H^{0}\left(k(X), \mathcal{H}^{2 n-1}\right)$, we may assume that $R^{a} \varphi_{*} \Omega_{Y / X}^{\bullet}$ is locally free and compatible with base change.

Via the diagram

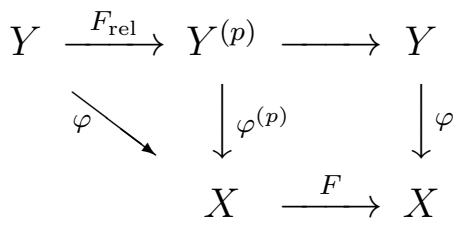

where $F$ is the absolute Frobenius of $X, \varphi^{(p)}=\varphi \times_{X} F, F_{\text {rel }}$ is the relative Frobenius, one knows by [15], (7.4) that the Gauß-Manin system $R^{a} \varphi_{*} \Omega_{Y / X}^{\bullet}$ has a Gauß-Manin stable filtration $R^{a} \varphi_{*}^{(p)} F_{\text {rel }}\left(\tau_{\leq a} \Omega_{Y / X}^{\bullet}\right)$, such that the restriction of $\nabla$ to the graded pieces $F^{*} R^{a-i} \varphi_{*} \Omega_{Y / X}^{i}$ is the trivial connection.

In particular, the graded pieces are locally generated by flat sections and $A_{i}=0$. So by additivity of the classes $c_{i}(E, \nabla), w_{n}(E, \nabla)=$ $\theta_{n}(E, \nabla)=0$.

In particular, the classes $w_{n}$ ( Gauß-Manin) provide examples of classes $w \in H^{0}\left(X, \mathcal{H}^{2 n-1}\right)$ whose restriction modulo $p$ vanish for all but finitely many $p$. This should imply that $w=0$ according to [17].

\section{REFERENCES}

[1] Atiyah, M.: Complex analytic connections in fibre bundles, Trans. Am. Math. Soc. 85 (1956), 181-207

[2] Bloch, S., Ogus, A.: Gersten's conjecture and the homology of schemes, Ann. Sc. ENS, $4^{0}$ série 7 (1974), 181 - 202

[3] Chern, S.-S.; Simons, J.: Characteristic forms and geometric invariants, Ann. of Math. 99 (1974), 48-68

[4] Corlette; K., Esnault, H.: Classes of local systems of $\mathbb{Q}$ hermitian vector spaces, preprint (1995)

[5] Deligne, P.: Équations Différentielles à Points Singuliers Réguliers, Springer Lecture Notes 163 (1970)

[6] Deligne, P.: Théorie de Hodge II, Publ. Math. IHES 40 (1972), 5 - 57

[7] Esnault, H.: Characteristic classes of flat bundles, Topology 27 (1988), 323 352 
[8] Esnault, H.: Characteristic classes of flat bundles, II, $K$-Theory 6 (1992), 45 $-56$

[9] Esnault, H.: Coniveau of Classes of Flat Bundles trivialized on a Finite Smooth Covering of a Complex Manifold, K-Theory 8 (1994), 483 - 497

[10] Esnault, H., Viehweg, E.: Logarithmic De Rham complexes and vanishing theorems, Inv. math. 86 (1986), 161 - 194

[11] Grothendieck, A.: EGA IV, Publ. Math. I.H.E.S. No.20 (1964)

[12] van der Geer, G.: private communication

[13] Hu, S.: Homotopy Theory, Academic Press (1959)

[14] Jouanolou, J.-P.: Une suite exacte de Mayer-Vietoris en $K$-théorie algébrique,in Algebraic K-theory I, Springer Lecture Notes 341, SpringerVerlag, (1973)

[15] Katz, N.: Nilpotent connections and the monodromy theorem: applications of a result of Turritin, Publ. Math. IHES 39 (1970), 175 - 232

[16] Mumford, D.: Towards an Enumerative Geometry of the Moduli Space of Curves, in Arithmetic and Geometry, volume II, Papers dedicated to I. R. Shafarevich, Birkhäuser, Progress in Mathematics 36 (1983), 271 - 327

[17] Ogus, A.: Differentials of the second kind and the conjugate spectral sequence (preprint)

[18] Reznikov, A.: All regulators of flat bundles are flat, Annals of Math. 139 (1994), 1 - 14

[19] Simpson, C.: Higgs bundles and local systems, Publ. Math. IHES 75 (1992), $5-95$

[20] Witten, E.: Quantum field theory and the Jones polynomial, Comm. Math. Phys. 121 (1989), 351 - 399

Department of Mathematics, University of Chicago, Chicago, IL 60637

E-mail address: bloch@math.uchicago.edu

Universität Essen,FB 6, Mathematik, 45117 Essen, Germany

E-mail address: esnault@uni-essen.de 